\title{
EXCLUDING INFINITE TREES
}

\author{
P. D. SEYMOUR AND ROBIN THOMAS
}

\begin{abstract}
For each infinite cardinal $\kappa$ we give several necessary and sufficient conditions for a graph not to contain a minor isomorphic to the infinite $\kappa$ branching tree in terms of a certain kind of a "tree-decomposition," in terms of a "path-decomposition," and also in terms of a "cops-and-robber game." We also give necessary and sufficient conditions for a graph not to contain a subgraph isomorphic to a subdivision of the same tree.
\end{abstract}

\section{INTRODUCTION}

In this paper graphs may be infinite, and may have loops and multiple edges. Let $G$ be a graph, let $\left\{G_{\alpha}\right\}_{\alpha \in \Lambda}$ be a collection of mutually vertex-disjoint connected nonnull subgraphs of $G$, and let $E$ be a set of edges of $G$ such that every member of $E$ has both its endpoints in $\bigcup_{\alpha \in \Lambda} G_{\alpha}$, but does not belong to $\bigcup_{\alpha \in \Lambda} G_{\alpha}$. The graph $H$ with vertex-set $\Lambda$ and edge-set $E$ with the obvious incidences is called a minor of $G$. The graphs $G_{\alpha}$ will be called the nodes of the minor. A graph $G$ is a subdivision of a graph $H$ if $G$ can be obtained from $H$ by replacing the edges of $H$ by internally disjoint paths joining the same ends.

For a cardinal $\kappa$ we define $T_{\kappa}$ to be the tree whose vertices are finite sequences (including the empty one) of ordinals $<\kappa$ with $\left(\alpha_{1}, \ldots, \alpha_{n}\right)$ and $\left(\beta_{1}, \ldots, \beta_{m}\right)$ being adjacent if $|n-m|=1$ and $\alpha_{i}=\beta_{i}$ for $i=1, \ldots$, $\min (n, m)$. Thus, if $\kappa$ is infinite then $T_{\kappa}$ is a regular tree of valency $\kappa$. (This is false in the finite case; for instance, $T_{2}$ is the tree with all vertices of valency 3 except for one of valency 2 .)

In $[1,5]$ it was proved that every finite graph with no minor isomorphic to a given finite tree admits a "path-decomposition" of bounded "width." In this paper we shall study some infinite extensions of this theorem, concerned with excluding $T_{\kappa}$ either as a minor or as a subdivision. It turns out that there are several relatively easy necessary and sufficient conditions, and we begin by introducing them.

The following is an extension of the cops-and-robber game from [8] to infinite cardinals. The game is played by two players on a graph $G$, with a fixed cardinal $\kappa$. One player controls the robber, and the other the cops. The cops attempt to catch the robber, and the robber to survive uncaptured. The robber stands on a vertex of the graph, and can at any time run at great speed to any other vertex

Received by the editors September 13,1989 and, in revised form, May 23, 1990 and October 22, 1990.

1991 Mathematics Subject Classification. Primary 05C05, 05 C75. 
along a path of the graph. He is not permitted to run through a cop, however. At any time there are any number $<\kappa$ cops, each of whom at any time either stands on a vertex or is in a helicopter (that is, is temporarily removed from the game). A move for the cop player consists either of having a set of cops take off or of "creating" more cops (subject to the restriction that the total is less than $\kappa$ ) and landing them on the vertices of the graph. The objective of the player controlling the movement of the cops is to land a cop via helicopter on the vertex occupied by the robber, and the robber's objective is to elude capture. (The point of the helicopters is that cops are not constrained to move along paths of the graph-they move from vertex to vertex arbitrarily.) The robber can see the helicopter approaching its landing spot and may run to a new vertex before the helicopter actually lands. In our version of the game the robber is visible and also can see the cops, so it is a full-knowledge game. It is also important that the cops win only if they capture the robber in finitely many steps. For extensions of this game see $\S 6$.

Let us make some definitions. We denote by $G \backslash X$ the graph obtained from $G$ by deleting $X$ (here $X$ may be a vertex or an edge, or a set of vertices or edges). If $X \subseteq V(G)$, the vertex set of a component of $G \backslash X$ is called an $X$-flap. We denote by $[V]^{<\kappa}$ the set of all subsets of $V$ of cardinality $<\kappa$.

Now we can state the game more precisely. A position is a pair $(X, R)$, where $X \in[V(G)]^{<\kappa}$ and $R$ is an $X$-flap. ( $X$ is the set of vertices currently occupied by cops, and $R$ tells us where the robber is - since he can run arbitrarily fast, all that matters is which component of $G \backslash X$ contains him.) We set $\left(X_{0}, R_{0}\right)$ to an initial position. In the normal game, $X_{0}=\varnothing$ and the robber chooses $R_{0}$ to be some component of $G$; however, in the analysis it will be useful to consider other initial positions. Now step 1 of the game begins. In general, at the start of the $i$ th step we have a position $\left(X_{i-1}, R_{i-1}\right)$. The cop player chooses a new set $X_{i} \in[V(G)]^{<\kappa}$ such that either $X_{i-1} \subseteq X_{i}$ or $X_{i} \subseteq X_{i-1}$. Then the robber player chooses (if possible) an $X_{i}$-flap $R_{i}$ satisfying $R_{i} \subseteq R_{i-1}$ or $R_{i-1} \subseteq R_{i}$ respectively. If this choice is impossible, that is, if $V\left(R_{i-1}\right) \subseteq X_{i}$, the cop player has won, and otherwise the game continues with step $i+1$. The robber player thus cannot win; his objective is to stop the cop player winning. If there is a winning strategy for the cop player, we say that " $<\kappa$ cops can search the graph."

To describe a strategy for the robber, we need the following definition. Let $G$ be a graph and $\kappa$ a cardinal. An escape of order $\kappa$ in $G$ is a function $\sigma$ which assigns to each $X \in[V(G)]^{<\kappa}$ the union of a set of $X$-flaps in such a way that

(i) if $X \subseteq Y$ then $\sigma(Y) \subseteq \sigma(X)$,

(ii) if $X \subseteq Y$ then every $X$-flap in $\sigma(X)$ intersects $\sigma(Y)$, and

(iii) $\sigma(\varnothing) \neq \varnothing$.

We speak of (i), (ii) and (iii) as the first, second, and third escape axioms. We remark that if (i) and (ii) are satisfied, then either $\sigma(X)=\varnothing$ for all $X \in[V(G)]^{<\kappa}$, or $\sigma(X) \neq \varnothing$ for all $X \in[V(G)]^{<\kappa}$, and so the third axiom ensures the latter. The relation to the cops-and-robber game is described in the following.

(1.1) The graph $G$ cannot be searched by $<\kappa$ cops if and only if there is an escape of order $\kappa$ in $G$. 
Proof. If there is an escape $\sigma$ of order $\kappa$, the robber can remain uncaptured by choosing $R_{i} \subseteq \sigma\left(X_{i}\right)$ at each step. Conversely, suppose that $\kappa$ cops cannot search the graph $G$. For each $X \in[V(G)]^{<\kappa}$, let $\sigma(X)$ be the union of all $X$-flaps $R$ such that the cop player cannot guarantee to win when the initial position is set to be $(X, R)$. Then $\sigma$ is an escape of order $\kappa$ in $G$.

So we see that escapes correspond to nonlosing strategies for the robber. In contrast to escapes of finite order in finite graphs (see [8]), escapes of infinite order in connected graphs are determined by trees, as follows. For $\kappa \geq \aleph_{0}$, a tree $T$ is called $\kappa$-balanced if $|E(T)| \geq 1$ and for every edge $e$ of $T$, both components of $T \backslash e$ have $\geq \kappa$ vertices, unless $\kappa=\aleph_{0}$ in which case $T$ is also permitted to be a one-way infinite path.

(1.2) Let $G$ be a connected graph and $\kappa$ an infinite cardinal. If $T$ is a subgraph of $G$ which is a $\kappa$-balanced tree, then there exists a unique escape $\sigma$ in $G$ of order $\kappa$ such that

$$
\text { for every } X \in[V(G)]^{<\kappa} \text { and every } X \text {-flap } C, C \subseteq \sigma(X) \text { if and }
$$
only if $|C \cap V(T)| \geq \kappa$.

Conversely, for every escape $\sigma$ of order $\kappa$ in $G$ there exists a subgraph $T$ of $G$ which is a $\kappa$-balanced tree and such that $(*)$ holds.

Our first equivalent condition will be in terms of escapes. The second will be in terms of "tree-decompositions." If $T$ is a tree and $t_{1}, t_{2} \in V(T)$, we denote by $T\left[t_{1}, t_{2}\right]$ the set of vertices of $T$ which lie on the path in $T$ between $t_{1}$ and $t_{2}$. A tree-decomposition of a graph $G$ is a pair $(T, W)$, where $T$ is a tree and $W=\left(W_{t}: t \in V(T)\right)$ is such that

(W1) $\bigcup_{t \in V(T)} W_{t}=V(G)$, and every edge of $G$ has both ends in some $W_{t}$, (W2) if $t^{\prime} \in T\left[t, t^{\prime \prime}\right]$, then $W_{t} \cap W_{t^{\prime \prime}} \subseteq W_{t^{\prime}}$.

Let $\kappa$ be a cardinal. A tree-decomposition $(T, W)$ has width $<\kappa$ if $\left|W_{t}\right|<\kappa$ for every $t \in V(T)$ and $\left|\bigcup_{i=1}^{\infty} \bigcap_{j \geq i} W_{t_{j}}\right|<\kappa$ for every infinite path $t_{1}, t_{2}, \ldots$ in $T$. It has adhesion $<\kappa$ if $\left|\bar{W}_{t} \cap W_{t^{\prime}}\right|<\kappa$ for every $\left\{t, t^{\prime}\right\} \in E(T)$ and $\liminf \operatorname{in}_{i \rightarrow \infty}\left|W_{t_{i}} \cap W_{t_{i+1}}\right|<\kappa$ for every infinite path $t_{1}, t_{2}, \ldots$ in $T$. Unfortunately, we also need a more general type of "tree-decomposition," but we postpone its definition until it is needed.

The third equivalent condition will be in terms of an analogue of the "pathdecompositions" of $[1,5]$. A well-ordered decomposition of a graph $G$ is a pair $(\lambda, W)$, where $\lambda$ is an ordinal and $W=\left(W_{\alpha}: \alpha \leq \lambda\right)$ is such that

(WO1) $\bigcup_{\alpha \leq \lambda} W_{\alpha}=V(G)$, and every edge of $G$ has both ends in $W_{\alpha}$ for some $\alpha \leq \lambda$

(WO2) if $\alpha \leq \beta \leq \gamma \leq \lambda$, then $W_{\alpha} \cap W_{\gamma} \subseteq W_{\beta}$, and

(WO3) if $\alpha \leq \lambda$ is a limit ordinal, then for all $\beta<\alpha, \bigcap_{\alpha>\gamma>\beta} W_{\gamma} \subseteq W_{\alpha}$.

We say that $(\lambda, W)$ has width $<\kappa$ if $\left|W_{\alpha}\right|<\kappa$ for every $\alpha \leq \lambda$. It has adhesion $<\kappa$ if for every ordinal $\alpha \leq \lambda$ there exists a cardinal $\kappa^{\prime}<\kappa$ such that for every $\beta<\alpha$ there exists $\gamma$ such that $\beta \leq \gamma<\alpha$ and $\left|W_{\gamma} \cap W_{\gamma+1}\right| \leq \kappa^{\prime}$, and if $\alpha<\lambda$ then $\left|W_{\alpha} \cap W_{\alpha+1}\right| \leq \kappa^{\prime}$. 
A ray is a one-way infinite path and a graph is called rayless if it has no ray. A tree-decomposition $(T, W)$ is called rayless if $T$ is rayless. Rayless trees are easy to handle-they admit an "ordinal ranking."

Now we can state our results in preliminary forms; full versions are stated later.

(1.3) For a graph $G$ and an uncountable cardinal $\kappa$, the following conditions are equivalent:

(i) $G$ has no minor isomorphic to $T_{\kappa}$,

(ii) $G$ has no escape of order $\kappa$,

(iii) $<\kappa$ cops can search $G$,

(iv) $G$ admits a rayless tree-decomposition of width $<\kappa$,

(v) $G$ admits a well-ordered decomposition of width $<\kappa$ and adhesion $<\kappa$.

For $\kappa=\aleph_{0}$, the analogue of (1.3) is the following. (The equivalence of (i) and (iv) in (1.4) was proved by Halin [2].)

(1.4) For a graph $G$, the following conditions are equivalent:

(i) $G$ is rayless,

(ii) $G$ has no escape of order $\aleph_{0}$.

(iii) $<\aleph_{0}$ cops can search $G$,

(iv) $G$ admits a rayless tree-decomposition of width $<\aleph_{0}$.

In order to characterize graphs with no minor isomorphic to $T_{\aleph_{0}}$, we need the following definitions. Let $\sigma$ be an escape of order $\kappa$ in a graph $G$. A subset $Y \in[V(G)]^{<\kappa}$ is said to be later than a subset $X \in[V(G)]^{<\kappa}$ if $|Y| \leq|X|$, $Y \subseteq X \cup \sigma(X)$, and $\sigma(Y) \subseteq \sigma(X)$. A subset $X \in[V(G)]^{<\kappa}$ is said to be terminal if there is no later $Y \subseteq V(G)$ with $Y \neq X$. The escape $\sigma$ is said to be massive if for every $X \in[V(G)]^{<\kappa}$ there is a later set $Y$ which is terminal.

A tree $T$ is called scattered if it contains no subgraph isomorphic to a subdivision of $T_{2}$, and a tree-decomposition $(T, W)$ is called scattered if $T$ is scattered. Scattered trees are well understood-see (4.5) for a result of [4].

(1.5) For a graph $G$, the following conditions are equivalent:

(i) $G$ contains no minor isomorphic to $T_{\aleph_{0}}$,

(ii) $G$ contains no minor isomorphic to $T_{2}$,

(iii) $G$ contains no subgraph isomorphic to a subdivision of $T_{2}$,

(iv) $G$ has no massive escape of order $\aleph_{0}$,

(v) $G$ admits a scattered tree-decomposition of width $<\aleph_{0}$ and adhesion $<\aleph_{0}$,

(vi) $G$ admits a well-ordered decomposition of width $<\aleph_{0}$ and adhesion $<\aleph_{0}$.

An escape $\sigma$ of order $\kappa$ in a graph $G$ is called major if for every $X \in$ $[V(G)]^{<\kappa}$ and every $X$-flap $C \subseteq \sigma(X)$ there exists a vertex $v \in C$ such that $v \in Y \cup \sigma(Y)$ for every $Y \in[V(G)]^{<\kappa}$.

We state only a special case of our last result; the full version is presented in $\S 4$. (For a definition of $\operatorname{cf}(\kappa)$ see the end of this section.)

(1.6) For a graph $G$ and an infinite cardinal $\kappa$, the following conditions are equivalent:

(i) $G$ contains no subgraph isomorphic to a subdivision of $T_{\kappa}$, 
(ii) $G$ has no major escape of order $\kappa$,

(iii) $G$ admits a well-ordered decomposition of width $<\kappa$.

If $\operatorname{cf}(\kappa)=\omega$, then these conditions are moreover equivalent to

(iv) $G$ admits a scattered tree-decomposition of width $<\kappa$.

We remark that (iii) $\Rightarrow$ (iv) of (1.6) is false in general. For example, let $\kappa$ be a singular cardinal with $\operatorname{cf}(\kappa)$ uncountable, let $\kappa=\sup \left\{\kappa_{\alpha}: \alpha<\operatorname{cf}(\kappa)\right\}$, where every $\kappa_{\alpha}<\kappa$, and let $G$ be the graph whose vertices are all pairs of ordinals $(\alpha, \beta)$ such that $\alpha<\operatorname{cf}(\kappa)$ and $\beta<\kappa_{\alpha}$, with $(\alpha, \beta)$ and $\left(\alpha^{\prime}, \beta^{\prime}\right)$ being adjacent if and only if either $\alpha=\alpha^{\prime}$ or $\beta=\beta^{\prime}$. It can be verified that $G$ admits a well-ordered decomposition of width $<\kappa$, but does not admit a treedecomposition of width $<\kappa$. A condition similar to (iv) which is equivalent to the other three conditions of $(1.6)$ is formulated in $\S 4$. Let us also remark that if $\kappa$ is a regular uncountable cardinal, then the conditions of (1.3) are equivalent to those of (1.6) (because in that case (2.3)(ii) $\Rightarrow(1.6)(\mathrm{i}) \Rightarrow(2.3)(\mathrm{iii})$ ).

All results presented so far hold in ZFC. In $\S 7$ we discuss a structure theorem which is less interesting from the point of view of graph theory, but which is undecidable in ZFC.

The paper is organized as follows. In $\S 2$ we prove (1.3); in $\S 3$ we prove (1.2), (1.4), and (1.5); in $\S 4$ we prove (1.6); in $\S 5$ we investigate the structure of the set of all escapes; in $\S 6$ we are concerned with modifications of the cops-and-robber game; and finally $\S 7$ contains the undecidability result.

Our notation about ordinals and cardinals is standard. We identify each ordinal with the set of all its predecessors, and each cardinal is also treated as an ordinal. Let $\lambda$ be an ordinal. A set $M \subseteq \lambda$ is said to be cofinal in $\lambda$ if for every $\alpha<\lambda$ there exists $\beta \in M$ such that $\alpha \leq \beta$. The least ordinal type of a set cofinal in $\lambda$ is called the cofinality of $\lambda$ and is denoted by $\operatorname{cf}(\lambda)$. An ordinal $\lambda$ is regular if $\operatorname{cf}(\lambda)=\lambda$, and otherwise it is singular. For a cardinal $\kappa$, we denote by $\kappa^{+}$the least cardinal $>\kappa$.

\section{ESCAPES OF UNCOUNTABLE ORDER}

In this section we prove (1.3), but before we do so we state and prove a result about escapes of any infinite order.

(2.1) For a graph $G$ and an infinite cardinal $\kappa$, the following conditions are equivalent.

(i) $G$ has no escape of order $\kappa$,

(ii) $G$ admits a rayless tree-decomposition of width $<\kappa$,

(iii) $<\kappa$ cops can search $G$.

Proof. We prove that (i) $\Rightarrow$ (ii) $\Rightarrow$ (iii); (iii) $\Rightarrow$ (i) follows from (1.1). Assume that $G$ has no escape of order $\kappa$. For $X \in[V(G)]^{<\kappa}$ let $\sigma(X)$ be the union of all $X$-flaps $C$ such that the restriction of $G$ to $C$ does not have a rayless tree-decomposition of width $<\kappa$.

(1) Let $X \in[V(G)]^{<\kappa}$, let $X^{\prime} \subseteq X$, and let $\left\{F_{\alpha}\right\}_{\alpha \in \Lambda}$ be a set of $X$-flaps, each disjoint from $\sigma(X)$. Then the subgraph $H$ of $G$ induced by $X^{\prime} \cup \bigcup_{\alpha \in \Lambda} F_{\alpha}$ admits a rayless tree-decomposition of width $<\kappa$. 
For let $X, X^{\prime},\left\{F_{\alpha}\right\}_{\alpha \in \Lambda}$, and $H$ be as stated, let $\left(T^{\alpha}, W^{\alpha}\right)$ be a rayless tree-decomposition of width $<\kappa$ of the restriction of $G$ to $F_{\alpha}(\alpha \in \Lambda)$, and let $W^{\alpha}=\left(W_{t}^{\alpha}: t \in V\left(T^{\alpha}\right)\right)$. Let $t_{0} \notin \bigcup_{\alpha \in \Lambda} V\left(T^{\alpha}\right)$ and let $T$ be a tree with vertex set $\bigcup_{\alpha \in \Lambda} V\left(T^{\alpha}\right) \cup\left\{t_{0}\right\}$ which contains every edge from $E\left(T^{\alpha}\right)$ for every $\alpha \in \Lambda$, and in which $t_{0}$ is adjacent to exactly one vertex of each $T^{\alpha}(\alpha \in \Lambda)$. We put

$$
W_{t}= \begin{cases}X^{\prime} \cup W_{t}^{\alpha} & \text { if } t \in V\left(T^{\alpha}\right), \\ X^{\prime} & \text { if } t=t_{0},\end{cases}
$$

and $W=\left(W_{t}: t \in V(T)\right)$. It follows easily that $(T, W)$ is a rayless treedecomposition of $H$ of width $<\kappa$, thus proving (1).

$\sigma$ satisfies the first two escape axioms.

For let $X \subseteq Y$ with $Y \in[V(G)]^{<\kappa}$, let $F$ be a $Y$-flap and let $F \subseteq K$, where $K$ is an $X$-flap, and assume that the restriction of $G$ to $K$ admits a rayless tree-decomposition $(T, W)$ of width $<\kappa$, where $W=\left(W_{t}: t \in V(T)\right)$. Let $W_{t}^{\prime}=W_{t} \cap F$ for $t \in V(T)$, and let $W^{\prime}=\left(W_{t}^{\prime}: t \in V(T)\right)$. Then $\left(T, W^{\prime}\right)$ is a rayless tree-decomposition of the restriction of $G$ to $F$ of width $<\kappa$. Thus $\sigma(Y) \subseteq \sigma(X)$, which verifies the first axiom.

For the second axiom, let $X \subseteq Y$ with $Y \in[V(G)]^{<\kappa}$ and let $K$ be an $X$-flap. Then $K=(K \cap Y) \cup \bigcup_{\alpha \in \Lambda} F_{\alpha}$, where $F_{\alpha}(\alpha \in \Lambda)$ are $Y$-flaps. If $K \subseteq \sigma(X)$ then by (1) (setting $X^{\prime}=K \cap Y$ ) there exists an $\alpha \in \Lambda$ such that $F_{\alpha} \subseteq \sigma(Y)$, which verifies the second axiom and hence proves (2).

It follows from (2) that $\sigma$ does not satisfy the third escape axiom. Hence every component of $G$ admits a rayless tree-decomposition of width $<\kappa$, and so $G$ itself admits such a decomposition by (1). Thus (i) $\Rightarrow$ (ii).

To prove that (ii) $\Rightarrow$ (iii) let $(T, W)$ be a rayless tree-decomposition of $G$ of width $<\kappa$. Here is a winning strategy for the cop player. Choose a vertex $t_{1} \in V(T)$, and let $X_{1}=W_{t_{1}}$. For $i \geq 2$, let the position at the start of the $i$ th move be $\left(X_{i-1}, R_{i-1}\right)$. The set $\left\{t \in V(T): R_{i-1} \cap W_{t} \neq \varnothing\right\}$ is the vertex set of a subtree of $T$, since $R_{i-1}$ is the vertex set of a connected subgraph of $G$; and so there is a vertex $t_{i} \in V(T)$ such that $R_{i-1} \cap W_{t_{i}} \neq \varnothing$, and $t_{i} \in T\left[t_{1}, t\right]$ for every $t \in V(T)$ such that $R_{i-1} \cap W_{t} \neq \varnothing$. Choose $X_{i}=X_{i-1} \cup W_{t_{i}}$. Then $R_{i}$ (if it exists) is a subset of $R_{i-1}$, and so $t_{i} \in T\left[t_{1}, t_{i+1}\right]$. Since this holds for all $i$ such that $R_{i}$ exists, we deduce that some $R_{i}$ does not exist, for otherwise $t_{1}, t_{2}, t_{3}, \ldots$ would all lie on some ray of $T$. Thus the cop player wins.

A linear decomposition of a graph $G$ is a pair $(L, W)$, where $L$ is a (Dedekind) complete linearly ordered set and $W=\left(W_{l}: l \in L\right)$ is such that

$$
\begin{aligned}
& \bigcup_{l \in L} W_{l}=V(G), \text { and every edge of } G \text { has both ends in some } W_{l}, \\
& \text { if } l \leq l^{\prime} \leq l^{\prime \prime}, \text { then } W_{l} \cap W_{l^{\prime \prime}} \subseteq W_{l^{\prime}}, \\
& \bigcap_{i \in I} W_{i} \subseteq W_{\inf (I)} \cap W_{\sup (I)} \text { for every nonempty interval } I \subseteq L .
\end{aligned}
$$

Let us remark that the requirement that $L$ be complete is not restrictive, because any "incomplete" decomposition can be completed in a natural way. Obviously, linear decompositions generalize well-ordered decompositions, in the sense that 
if $(\lambda, W)$ is a well-ordered decomposition then $(\lambda+1, W)$ is a linear decomposition. A linear decomposition $(L, W)$ has width $<\kappa$ if $\left|W_{l}\right|<\kappa$ for every $l \in L$. We say that $(L, W)$ has adhesion $\leq \kappa^{\prime}$ at $l \in L$ if for every $m \in L$ with $m \neq l$ there exist distinct $l_{1}, l_{2} \in L$ between $l$ and $m$ such that there is no $l_{3} \in L$ strictly between $l_{1}$ and $l_{2}$, and such that $\left|W_{l_{1}} \cap W_{l_{2}}\right| \leq \kappa^{\prime}$. We say that $(L, W)$ has adhesion $<\kappa$ if for every $l \in L$ there exists $\kappa^{\prime}<\kappa$ such that $(L, W)$ has adhesion $\leq \kappa^{\prime}$ at $l$.

A linear ordering $L$ is called scattered if the set of rational numbers cannot be monotonely embedded into $L$. We say that a linear decomposition $(L, W)$ of a graph $G$ is scattered linear if $L$ is scattered. We remark that we are using "scattered" in two different (but related) contexts: scattered trees and scattered linear orderings.

Let $\kappa$ be an infinite cardinal. We recall that a tree $T$ with $E(T) \neq \varnothing$ is $\kappa$-balanced if either for every edge $e$, both components of $T \backslash e$ have $\geq \kappa$ vertices, or $\kappa=\aleph_{0}$ and $T$ is a ray.

(2.2) Let $T$ be a tree with $E(T) \neq \varnothing$ which is not a ray. If $\kappa=\aleph_{0}, T$ is $\kappa$-balanced if and only if for every $e \in E(T)$, both components of $T \backslash e$ have a ray. For $\kappa \geq \aleph_{1}, T$ is $\kappa$-balanced if and only if for every $e \in E(T)$, both components of $T \backslash e$ have a $T_{\kappa}$-minor.

Proof. "If" is clear for all $\kappa$, and "only if" is clear for $\kappa=\aleph_{0}$. For $\kappa \geq \aleph_{1}$, let $T$ be $\kappa$-balanced, let $e_{0} \in E(T)$, and let $R$ be a component of $T \backslash e_{0}$. We shall construct a mapping $\eta$ which assigns to each $t \in V\left(T_{\kappa}\right)$ a subset of $V(R)$ and to each $e \in E\left(T_{\kappa}\right)$ an edge of $R$ in such a way that

$$
\eta(t) \text { induces a tree in } R \text { for every } t \in V\left(T_{\kappa}\right) \text {, }
$$$$
\text { if } t, t^{\prime} \in V\left(T_{\kappa}\right) \text { and } t \neq t^{\prime} \text {, then } \eta(t) \cap \eta\left(t^{\prime}\right)=\varnothing \text {, }
$$

if $t, t^{\prime} \in V\left(T_{\kappa}\right)$ and $e$ is an edge of $T_{\kappa}$ with endpoints $t, t^{\prime}$ then $\eta(e)$ has one endpoint in $\eta(t)$ and the other one in $\eta\left(t^{\prime}\right)$.

Let $\kappa=\sup \left(\kappa_{\alpha}: \alpha<\operatorname{cf}(\kappa)\right)$, where every $\kappa_{\alpha}$ is regular. We need the fact that every tree of cardinality $\geq \kappa$ contains a vertex of valency $\geq \kappa_{\alpha}$ for every $\alpha<\operatorname{cf}(\kappa)$. The mapping $\eta$ will be constructed in $\omega$ steps. In the first step we choose a vertex $r_{0} \in V(R)$ of valency $\geq \operatorname{cf}(\kappa)$, and let $\left\{C^{\alpha}\right\}_{\alpha<\mathrm{cf}(\kappa)}$ be some of the components of $T \backslash r_{0}$ contained in $R$. In each $C^{\alpha}$ choose a vertex $r^{\alpha}$ of valency $\geq \kappa_{\alpha}$, let $R_{0}$ be the smallest subtree of $R$ containing $r_{0}$ and all $r^{\alpha} \quad(\alpha<\operatorname{cf}(\kappa))$, and let $\eta$ of the null sequence be the vertex set of $R_{0}$. For $\alpha<\operatorname{cf}(\kappa)$ let $E_{\alpha} \subseteq E(R)$ be a set of edges of cardinality $\kappa_{\alpha}$, all incident with $r^{\alpha}$ but with no other vertex in $R_{0}$. Now if $e$ is an edge of $T_{\kappa}$ incident with the null sequence we define $\eta(e) \in \bigcup_{\alpha<\mathrm{cf}(\kappa)} E_{\alpha}$ arbitrarily subject to the requirement that $\eta$ be 1-1. This is clearly possible.

Now let $n \geq 0$ be an integer, let $M_{n}$ be the set of all $t \in V\left(T_{\kappa}\right)$ of the form $t=\left(\alpha_{1}, \ldots, \alpha_{k}\right)$ for $k \leq n$, and assume that we have defined $\eta(t)$ for all $t \in M_{n}$ and all edges incident to vertices in $M_{n}$ in such a way that (1)-(3) are satisfied for all $t, t^{\prime} \in M_{n}$, and

if $e$ is an edge of $T_{\kappa}$ with endpoints $t \in M_{n}$ and $t^{\prime} \in M_{n+1}$ $M_{n}$, then $\eta(e)$ has exactly one endpoint in $\eta(t)$, and $V\left(B_{e}\right) \subseteq$ $V(R)$, where $B_{e}$ is the component of $T \backslash e$ which is disjoint from $\eta(t)$. 
It follows that if $t, t^{\prime}, e$ are as in (4) then $V\left(B_{e}\right) \cap \eta\left(t^{\prime \prime}\right)=\varnothing$ for all $t^{\prime \prime} \in M_{n}$. We must define $\eta(t)$ for all $t \in M_{n+1}-M_{n}$ and $\eta(e)$ for all edges $e$ incident to vertices from $M_{n+1}-M_{n}$. To this end we fix $t=\left(\alpha_{1}, \ldots, \alpha_{n}\right) \in M_{n}$, and let $e$ be an edge of $T_{\kappa}$ with one endpoint $t$ and the other say $t_{e}=$ $\left(\alpha_{1}, \ldots, \alpha_{n}, \alpha_{e}\right) \in M_{n+1}$. Let $B_{e}$ be as in (4), and let $r_{e} \in V\left(B_{e}\right)$ be an endpoint of $\eta(e)$. We select $r_{e}^{\prime}$ in $B_{e}$ of valency $\geq \operatorname{cf}(\kappa)$, let $\left\{C_{e}^{\alpha}\right\}_{\alpha<\mathrm{cf}(\kappa)}$ be some of the components of $T \backslash r_{e}^{\prime}$ which are included in $V(R)$, and let $r_{e}^{\alpha}$ be a vertex of $C_{e}^{\alpha}$ of valency $\geq \kappa_{\alpha}$. We define $\eta\left(t_{e}\right)$ to be the vertex set of the minimal subtree of $R$ containing $r_{e}$ and all $r_{e}^{\alpha}(\alpha<\operatorname{cf}(\kappa))$. Let $E_{e}$ be a set of $\kappa$ edges with one endpoint some $r_{e}^{\alpha} \quad(\alpha<\operatorname{cf}(\kappa))$ and the other not in $\eta\left(t_{e}\right)$. If $f$ is an edge of $T_{\kappa}$ with one endpoint $t_{e}$ and the other in $M_{n+2}-M_{n+1}$ we define $\eta(f) \in E_{e}$ arbitrarily subject to the requirement that $\eta$ be 1-1. This completes the inductive definition. It is easily seen that conditions (1)-(4) are satisfied. By (1)-(3) the sets $\eta(t)$ form the nodes of a minor of $R$ isomorphic to $T_{\kappa}$ as desired.

A tree $T$ is called a $\kappa$-nova if it is $\kappa$-balanced and every vertex has valency $\leq \kappa$, with strict inequality if $\kappa$ is singular. Obviously $T_{\kappa}$ is a $\kappa$-nova for every regular cardinal $\kappa$, but $\kappa$-novas exist even for infinite singular cardinals. Indeed, if $\kappa$ is infinite singular we may choose cardinals $\left\{\kappa_{\alpha}: \alpha<\operatorname{cf}(\kappa)\right\}$ with $\kappa_{\alpha}<\kappa$ and $\sup \left\{\kappa_{\alpha}: \alpha<\operatorname{cf}(\kappa)\right\}=\kappa$ and consider the subtree of $T_{\kappa}$ consisting of all sequences $\left(\alpha_{1}, \ldots, \alpha_{n}\right)$, where $\alpha_{i}<\operatorname{cf}(\kappa)$ if $i$ is odd and $\alpha_{i}<\kappa_{\alpha_{i-1}}$ if $i$ is even. This tree is a $\kappa$-nova.

The following is an expanded form of (1.3).

(2.3) For a graph $G$ and an uncountable cardinal $\kappa$, the following conditions are equivalent:

(i) $G$ has no minor isomorphic to $T_{\kappa}$,

(ii) for every $\kappa$-nova $T, G$ contains no subgraph isomorphic to a subdivision of $T$,

(iii) for some $\kappa$-nova $T, G$ contains no subgraph isomorphic to a subdivision of $T$,

(iv) $G$ has no escape of order $\kappa$,

(v) $<\kappa$ cops can search $G$,

(vi) $G$ admits a rayless tree-decomposition of width $<\kappa$,

(vii) $G$ admits a scattered tree-decomposition of width $<\kappa$ and adhesion $<\kappa$,

(viii) $G$ admits a well-ordered decomposition of width $<\kappa$ and adhesion $<\kappa$,

(ix) $G$ admits a scattered linear decomposition of width $<\kappa$ and adhesion $<\kappa$.

Implications (vi) $\Rightarrow$ (vii) and (viii) $\Rightarrow$ (ix) are trivial, (i) $\Rightarrow$ (ii) follows from (2.2), (ii) $\Rightarrow$ (iii) follows since a $\kappa$-nova exists, (iv) $\Rightarrow$ (v) $\Rightarrow$ (vi) follows from (2.1), and (vii) $\Rightarrow$ (viii) follows from (4.6). In the rest of this section we prove that (iii) $\Rightarrow$ (iv) and (ix) $\Rightarrow$ (i).

We start with a lemma which will be used in this and a subsequent paper. Let $\sigma$ be an escape in a graph $G$ of order $\kappa \geq \aleph_{0}$ and let $\kappa^{\prime} \leq \kappa$. We say that a vertex $\nu \in V(G)$ is $\kappa^{\prime}$-major if $\nu \in X \cup \sigma(X)$ for every $X \in[V(G)]^{<\kappa^{\prime}}$ and we say that it is major if it is $\kappa$-major.

(2.4) Let $\sigma$ be an escape of order $\kappa \geq \aleph_{1}$ in a graph $G$ and let $\kappa^{\prime}$ be a regular 
cardinal with $\aleph_{0} \leq \kappa^{\prime} \leq \kappa$. Let $X \in[V(G)]^{<\kappa^{\prime}}$, and let $C$ be an $X$-flap with $C \subseteq \sigma(X)$. Then there exists a $\kappa^{\prime}$-major vertex in $C$.

Proof. For each $v \in V(G)$ which is not $\kappa^{\prime}$-major, there exists by definition a set $X_{v} \in[V(G)]^{<\kappa^{\prime}}$ with $v \notin X_{v} \cup \sigma\left(X_{v}\right)$. We construct a sequence $X_{0} \subseteq$ $X_{1} \subseteq X_{2} \subseteq \cdots$ of subsets of $V(G)$, each of cardinality $<\kappa^{\prime}$, as follows. Let $X_{0} \subseteq V(G)$ be any subset of $V(G)$ of size $<\kappa^{\prime}$ with $X_{0} \cap C \neq \varnothing$ and $X \subseteq X_{0}$. Assume that we have already constructed sets $X_{0} \subseteq \cdots \subseteq X_{n}$, and let us construct $X_{n+1}$. Let $X_{n+1}=X_{n} \cup \bigcup X_{v}$, the union taken over all $v \in X_{n}$ which are not $\kappa^{\prime}$-major. We see that $\left|X_{n+1}\right|<\kappa^{\prime}$ since $\kappa^{\prime}$ is regular.

Let $Y=\bigcup_{n=0}^{\infty} X_{n}$; then $|Y|<\kappa$ since $\kappa \geq \aleph_{1}$. Let us choose $u \in C \cap \sigma(Y)$, adjacent to a vertex $v \in C \cap Y$. (This is possible, for $C \cap Y \neq \varnothing$, and $C \cap \sigma(Y) \neq \varnothing$ from the second escape axiom.) We claim that $v$ is $\kappa^{\prime}$-major. For if not then $v \notin X_{v} \cup \sigma\left(X_{v}\right)$ and $X_{v} \subseteq Y$, and therefore $u \in \sigma(Y) \subseteq \sigma\left(X_{v}\right)$, which is impossible since $u, v$ are adjacent.

We recall that an escape $\sigma$ of order $\kappa$ in $G$ is major if for every $X \in$ $[V(G)]^{<\kappa}$ and for every $X$-flap $C \subseteq \sigma(X)$ there is a major vertex in $C$. From (2.4) we deduce that if $\kappa \geq \aleph_{1}$, and is regular, then every escape of order $\kappa$ is major.

(2.5) Let $\kappa$ be an infinite cardinal, and let $T$ be a tree on $\kappa$ vertices. Then there exists a well-ordering $\left\{t_{\alpha}\right\}_{\alpha<\kappa}$ of the vertices of $T$ of order type $\kappa$ such that

(i) for $\alpha>0, t_{\alpha}$ is adjacent to exactly one $t_{\beta}$ with $\beta<\alpha$, and

(ii) if $t \in V(T)$ has valency $<\kappa$, then $\left\{\alpha<\kappa: t_{\alpha}\right.$ is adjacent to $\left.t\right\}$ is not cofinal in $\kappa$.

Proof. We may assume that the vertices of $T$ are finite sequences of ordinals such that

(a) $\left(\beta_{1}, \ldots, \beta_{n}\right) \in V(T)$ and $\left(\beta_{1}^{\prime}, \ldots, \beta_{n^{\prime}}^{\prime}\right) \in V(T)$ are adjacent if and only if $\left|n-n^{\prime}\right|=1$ and $\beta_{i}=\beta_{i}^{\prime}$ for $i=1, \ldots, \min \left(n, n^{\prime}\right)$

(b) if $\left(\beta_{1}, \ldots, \beta_{n}\right) \in V(T)$ then $\beta_{i} \geq i$ for $1 \leq i \leq n$, and

(c) if $\left(\beta_{1}, \ldots, \beta_{n}\right) \in V(T)$ where $n \geq 1$ then $\left(\beta_{1}, \ldots, \beta_{n-1}\right) \in V(T)$, and if $n \leq \beta \leq \beta_{n}$ then $\left(\beta_{1}, \ldots, \beta_{n-1}, \beta\right) \in V(T)$.

Let $t_{0}$ be the empty sequence and assume that $\left\{t_{\beta}: \beta<\alpha\right\} \subseteq V(T)$ have already been defined. If $V(T)=\left\{t_{\beta}: \beta<\alpha\right\}$, we stop. Otherwise we choose $t_{\alpha}=\left(\beta_{1}, \ldots, \beta_{n}\right)$ in $V(T)-\left\{t_{\beta}: \beta<\alpha\right\}$ such that

$$
\max \left\{\beta_{1}, \ldots, \beta_{n}\right\} \text { is as small as possible, }
$$

and, subject to (1),

$n$ is as small as possible.

Condition (i) now follows easily.

If $\chi$ is an infinite cardinal and $\beta_{1}, \ldots, \beta_{n}<\chi$ are ordinals, and $t_{\alpha}=\left(\beta_{1}, \ldots, \beta_{n}\right) \in V(T)$ then $\alpha<\chi$.

For let $\beta=\max \left(\beta_{1}, \ldots, \beta_{n}\right)$. Since $\beta<\chi$, the set of finite sequences of ordinals $<\beta$ which belong to $V(T)$ has cardinality $<\chi$ (using (b) if $\chi=\aleph_{0}$ ). The claim follows. 
We deduce that $V(T)=\left\{t_{\alpha}: \alpha<\kappa\right\}$, since the existence of $t_{\kappa}$ would violate (3). It remains to prove (ii), and we assume that $\kappa$ is singular, because (ii) is clear when $\kappa$ is regular. Suppose that $t=\left(\beta_{1}, \ldots, \beta_{n}\right) \in V(T)$ has valency $\mu<\kappa$. Let $\lambda=\max \left(\left|\beta_{1}\right|, \ldots,\left|\beta_{n}\right|, \mu, \aleph_{0}\right)$; then $\lambda<\kappa$, and so $\lambda^{+}<\kappa$ since $\kappa$ is singular and hence not a successor cardinal. By (3), $\alpha<\lambda^{+}$for every $\alpha<\kappa$ such that $t_{\alpha}$ is adjacent to $t$, and hence (ii) follows, as required.

For a graph $G$, let $P(G)$ denote the set of finite (possibly closed) paths in $G$, containing at least one edge. A subgraph of $G$ is isomorphic to a subdivision of a graph $H$ if and only if there exists a mapping $h: V(H) \cup E(H) \rightarrow V(G) \cup P(G)$, called a homeomorphic embedding of $H$ into $G$, such that

(i) $h(V(H)) \subseteq V(G), h(E(H)) \subseteq P(G)$,

(ii) $h$ is injective,

(iii) if $e \in E(H)$ has end vertices $u, v \in V(H)$, then the path $h(e)$ joins $h(u)$ and $h(v)$ and uses no $h(w)$ for $w \in V(H)-\{u, v\}$,

(iv) if $e, e^{\prime}$ are distinct edges of $H$, then $h(e)$ and $h\left(e^{\prime}\right)$ are internally disjoint.

(2.6) Let $G$ be a graph, and let $\kappa, \mu$ be infinite cardinals with $\mu \geq \aleph_{1}$ and $\mu=\kappa$ or $\kappa^{+}$and let $\sigma$ be an escape of order $\kappa$ in $G$ such that for every $\mu^{\prime}<\mu$, every $X \in[V(G)]^{<\mu^{\prime}}$ and every $X$-flap $C \subseteq \sigma(X)$ there exists a $\mu^{\prime}$ major vertex in $C$. Let $T$ be a tree with $|V(T)|=\kappa$ and with every vertex of valency $<\mu$, and let $l$ be a function from $V(T)$ to the set of cardinals $<\mu$. Then there exists a homeomorphic embedding $h$ of $T$ into $G$ such that $h(t)$ is $l(t)$-major for every $t \in V(T)$.

Proof. Since $|V(T)|=\kappa$, every vertex of $T$ has valency $<\mu$ and $\mu \geq \aleph_{1}$, it follows that if $\mu=\kappa$ then $\kappa$ is singular. Let $\left\{t_{\alpha}\right\}_{\alpha<\kappa}$ be the well ordering of $V(T)$ as in (2.5). For each $t \in V(T)$, if $\mu=\kappa^{+}$let $\lambda(t)=\kappa$, and if $\mu=\kappa$ let $\lambda(t)<\kappa$ be an infinite cardinal such that $l(t) \leq \lambda(t)$ and $\alpha<\lambda(t)$ for every $t_{\alpha} \in V(T)$ adjacent to $t$. (This exists since $\kappa$ is singular and the well-ordering satisfies (2.5ii).) If $\alpha \leq \kappa$ is an ordinal, we denote by $S_{\alpha}$ the subtree of $T$ consisting of all vertices $t_{\beta}$ for $\beta<\alpha$. A majority is a pair $(\alpha, h)$, where $\alpha \leq \kappa$ is an ordinal and $h$ is a homeomorphic embedding of $S_{\alpha}$ into $G$ such that

(1) for every $t \in V\left(S_{\alpha}\right), h(t)$ is $\lambda(t)$-major.

If $(\alpha, h),\left(\alpha^{\prime}, h^{\prime}\right)$ are two majorities, we put $(\alpha, h) \leq\left(\alpha^{\prime}, h^{\prime}\right)$ if $\alpha \leq \alpha^{\prime}$ and $h^{\prime}$ restricted to $S_{\alpha}$ coincides with $h$. It follows from Zorn's Lemma that there exists a maximal majority $(\alpha, h)$. We claim that $\alpha=\kappa$, which will complete the proof. So suppose for a contradiction that $\alpha<\kappa$ and let $X$ be the set of all vertices of the form $h(t)$ for $t \in V\left(S_{\alpha}\right)$ together with all vertices of the paths $h(e)$ for $e \in E\left(S_{\alpha}\right)$. By (2.5i), since $\alpha>0$ there exists $\beta<\alpha$ such that $t_{\alpha}$ is adjacent to $t_{\beta}$, and it follows that $|X|<\lambda\left(t_{\beta}\right)$ (because $X$ is finite if $\alpha$ is finite, and $|X| \leq|\alpha|<\lambda\left(t_{\beta}\right)$ if $\alpha$ is infinite).

There exists an $X$-flap $C \subseteq \sigma(X)$ such that $h\left(t_{\beta}\right)$ is adjacent to a vertex in $C$.

For otherwise $h\left(t_{\beta}\right) \notin \sigma\left(X-\left\{h\left(t_{\beta}\right)\right\}\right)$, a contradiction since $h\left(t_{\beta}\right)$ is $\lambda\left(t_{\beta}\right)$ major by (1). 
Now take a $\lambda\left(t_{\alpha}\right)$-major vertex $v \in C$, and take a path $P$ joining $v$ and $h\left(t_{\beta}\right)$ such that $P \backslash h\left(t_{\beta}\right)$ is contained in $C$. Put $\alpha^{\prime}=\alpha+1$, and let

$$
h^{\prime}(x)= \begin{cases}h(x) & \text { if } x \in V\left(S_{\alpha}\right) \cup E\left(S_{\alpha}\right), \\ v & \text { if } x=t_{\alpha}, \\ P & \text { if } x \text { is the edge of } S_{\alpha+1} \text { joining } t_{\alpha} \text { and } t_{\beta} .\end{cases}
$$

Then $(\alpha, h)<\left(\alpha^{\prime}, h^{\prime}\right)$, a contradiction.

Let $G$ be a graph and $\sigma$ an escape in $G$ of order $\kappa$. We say that a connected subgraph $H$ of $G$ is major if $V(H) \cap(X \cup \sigma(X)) \neq \varnothing$ for every $X \in[V(G)]^{<\kappa}$. The following corollary implies (iii) $\Rightarrow$ (iv) in (2.3).

(2.7) Let $G$ be a graph, let $\sigma$ be an escape in $G$ of order $\kappa \geq \aleph_{1}$, and let $T$ be a $\kappa$-nova. Then there exists a subgraph $S$ of $G$ isomorphic to a subdivision of $T$ such that for every edge $e$ of $S$, both components of $S \backslash e$ are major subgraphs.

Proof. Let $\mu=\kappa^{+}$if $\kappa$ is regular, and $\mu=\kappa$ otherwise. For each $t \in V(T)$, let $l(t)$ be the valency of $t$ in $T$. By (2.4) and (2.6), there is a subgraph $S$ of $G$ isomorphic to a subdivision of $T$, such that for each $t \in V(T)$, the corresponding vertex $s \in V(S)$ is $l(t)$-major. For each regular cardinal $\kappa^{\prime} \leq \kappa$, since for each edge $f$ of $T$ both components of $T \backslash f$ have vertices of valency $\geq \kappa^{\prime}$, it follows that for each edge $e$ of $S$ both components of $S \backslash e$ have $\kappa^{\prime}$ major vertices; and hence both components of $S \backslash e$ are major, as required.

In the next section we shall need the following variation of (2.7).

(2.8) Let $G$ be a graph, let $\sigma$ be an escape in $G$ of order $\kappa \geq \aleph_{1}$, let $Z \in$ $[V(G)]^{<\kappa}$ and let $H$ be a component of $G \backslash Z$ with $V(H) \subseteq \sigma(Z)$. Then there exists a subgraph $T$ of $H$, which is a $\kappa$-nova, such that for every edge e of $T$, both components of $T \backslash e$ are major.

Proof. We define $\tau(X)=\sigma(X \cup Z) \cap V(H)$, for $X \in[V(H)]^{<\kappa}$. It is easily seen that $\tau$ is an escape of order $\kappa$ in $H$ and thus the result follows from (2.7), for any subdivision of a $\kappa$-nova is a $\kappa$-nova.

Now we begin the proof that (ix) $\Rightarrow(i)$ in $(2.3)$.

(2.9) Let $(L, W)$ be a linear decomposition of a graph $G$, let $H$ be a connected subgraph of $G$ and let $l_{1}, l_{2}, l_{3}, l_{4} \in L$ be such that $l_{1}<l_{2} \leq l_{3}<l_{4}$, there is no $l \in L$ with $l_{2}<l<l_{3}$, and $V(H) \cap W_{l_{1}} \neq \varnothing \neq V(H) \cap W_{l_{4}}$. Then $V(H) \cap W_{l_{2}} \cap W_{l_{3}} \neq \varnothing$.

Proof. Suppose not. Then (using (L2)) there exist adjacent vertices $u, v \in$ $V(H)$ such that $u \in \bigcup_{l \leq l_{2}} W_{l}-\bigcup_{l \geq l_{3}} W_{l}$ and $v \in \bigcup_{l \geq l_{3}} W_{l}-\bigcup_{l \leq l_{2}} W_{l}$. By (L1) there exists $l \in L$ with $u, v \in W_{l}$, and hence $l_{2}<l<l_{3}$, a contradiction.

(2.10) Let $\kappa$ be an infinite cardinal, let $(L, W)$ be a linear decomposition of width $<\kappa$ of a graph $G$, and let $H$ be a minor of $G$. Assume furthermore that either

(i) every node of the minor has $<\mathrm{cf}(\kappa)$ vertices, or

(ii) $\kappa$ is a regular uncountable cardinal, or

(iii) $(L, W)$ has adhesion $<\kappa$. 
Then there exists a linear decomposition $(L, U)$ (that is, using the same linear ordering) of $H$ of width $<\kappa$.

Proof. We say that $l \in L$ has a predecessor if there exists $l^{\prime}<l$ such that there is no $l^{\prime \prime} \in L$ with $l^{\prime}<l^{\prime \prime}<l$, and we say that it has a successor if it has a predecessor in the inverse linear ordering. Let $\left\{G_{\alpha}\right\}_{\alpha \in \Lambda}$ be the nodes of $H$. For $l \in L$, we define

$$
\begin{gathered}
V_{l}=\left\{\alpha \in \Lambda: V\left(G_{\alpha}\right) \cap W_{l} \neq \varnothing\right\}, \\
U_{l}^{-}= \begin{cases}\varnothing & \text { if } l \text { has a predecessor, } \\
\bigcup_{l^{\prime}<l} \cap_{l^{\prime}<l^{\prime \prime}<l} V_{l^{\prime \prime}} & \text { otherwise, }\end{cases} \\
U_{l}^{+}= \begin{cases}\varnothing & \text { if } l \text { has a successor, } \\
\bigcup_{l^{\prime}>l} \cap_{l^{\prime}>l^{\prime \prime}>l} V_{l^{\prime \prime}} & \text { otherwise, } \\
U_{l}=U_{l}^{-} \cup V_{l} \cup U_{l}^{+} .\end{cases}
\end{gathered}
$$

Let $U=\left(U_{l}: l \in L\right)$. We claim that $(L, U)$ is a linear decomposition of $H$. Condition (L1) follows easily from the fact that $(L, W)$ satisfies (L1). To prove (L2) let $l_{1}, l_{2}, l \in L$ satisfy $l_{1}<l<l_{2}$, and let $\alpha \in U_{l_{1}} \cap U_{l_{2}}$. Then there exist $l_{1}^{\prime}, l_{2}^{\prime} \in L$ such that $l_{1}^{\prime}<l<l_{2}^{\prime}$ and $\alpha \in V_{l^{\prime}} \cap V_{l^{\prime}}$, and hence $\alpha \in V_{l} \subseteq U_{l}$ by (2.9). To prove (L3) let $\alpha \in \Lambda$ and let $I=\left\{l \in L: \alpha \in U_{l}\right\}$. By symmetry it is enough to prove that $\inf (I) \in I$. Suppose not and let $l_{0}=\inf (I)$. Since $l_{0} \notin I$ it follows that $l_{0}$ has no successor. Choose $l_{2} \in I$. We claim that $\alpha \in V_{l}$ for all $l \in I$ with $l \leq l_{2}$. For since $l$ is not the successor of $l_{0}$, there exists $l_{1} \in I$ with $l_{1}<l$; then $\alpha \in U_{l_{1}} \cap U_{l_{2}}$, and as in the proof of (L2) above it follows that $\alpha \in V_{l}$, as claimed. We deduce that $\alpha \in U_{l_{0}}^{+} \subseteq U_{l_{0}}$, a contradiction. This completes the proof of our claim that $(L, U)$ is a linear decomposition of $H$. It remains to check its width. Clearly $\left|V_{l}\right| \leq\left|W_{l}\right|<\kappa$. We must show that $\left|U_{l}^{-}\right|<\kappa$ and $\left|U_{l}^{+}\right|<\kappa$. We prove the former, for the latter follows by symmetry. Before we do so we need two definitions.

Let $l \in L$. We say that a set $M \subseteq L$ well-ordered by the ordering of $L$ is lower cofinal for $l$ if $m<l$ for every $m \in M$ and for every $l^{\prime} \in L$ with $l^{\prime}<l$ there exists an $m \in M$ such that $l^{\prime} \leq m$. It follows from Zorn's lemma that there exists a lower cofinal set for every $l \in L$. We define the lower cofinality of $l \in L$ to be the least ordinal $\alpha$ such that there exists a lower cofinal set for $l$ of order type $\alpha$. It follows from the standard proofs about cofinality that the lower cofinality is always either 0 (if $l=\inf (L))$ or 1 (if $l$ has a predecessor) or an infinite regular cardinal (otherwise).

Let $l \in L$. We may assume that $U_{l}^{-} \neq \varnothing$, and so $l \neq \inf (L)$ and $l$ has no predecessor.

(1) If the lower cofinality of $l \in L$ is smaller than $\operatorname{cf}(\kappa)$, then $\left|U_{l}^{-}\right|<\kappa$.

For let $M \subseteq L$ be a lower cofinal set for $l$ of cardinality $<\operatorname{cf}(\kappa)$. Then $U_{l}^{-} \subseteq \bigcup_{m \in M} V_{m}$, and hence $\left|U_{l}^{-}\right|<\kappa$.

In view of (1), we assume from now on that $l \in L$ is such that

(2) The lower cofinality of $l$ is at least $\mathrm{cf}(\kappa)$.

(3) If (i) holds then $U_{l}^{-} \subseteq V_{l}$. 
For let $\alpha \in U_{l}^{-}$. Since $\left|V\left(G_{\alpha}\right)\right|<\operatorname{cf}(\kappa)$ it follows from (2) that there exists $v \in V\left(G_{\alpha}\right)$ such that for each $m \in L$ with $m<l$ there exists $s \in L$ with $m \leq s<l$ and $v \in W_{s}$. By (L2), since $l \neq \inf (L)$, there exists $m_{0} \in L$ with $m_{0}<l$ such that $v \in W_{m}$ for every $m \in L$ with $m_{0} \leq m<l$. Hence $v \in W_{l}$ by (L3), since $l$ has no predecessor, and thus $\alpha \in V_{l}$, as desired.

$$
\text { If (ii) holds then } U_{l}^{-} \subseteq V_{l} \text {. }
$$

For suppose that $\alpha \in U_{l}^{-}-V_{l}$. Choose $m_{0} \in L$ with $m_{0}<l$ such that $W_{m} \cap V\left(G_{\alpha}\right) \neq \varnothing$ for all $m \in L$ with $m_{0} \leq m<l$, and assume that we have already constructed $m_{0}, \ldots, m_{n-1} \in L$ with $m_{0}<m_{1}<\cdots<m_{n-1}<l$. By (2), since $\operatorname{cf}(\kappa)=\kappa$ and $\left|W_{m_{n-1}}\right|<\kappa$ and $l$ has no predecessor, there exists $m_{n} \in L$ such that $m_{n-1}<m_{n}<l$ and $W_{m_{n-1}} \cap V\left(G_{\alpha}\right) \cap W_{m_{n}}=\varnothing$. This completes the construction of an infinite sequence $m_{0}<m_{1}<\cdots$ of elements of $L$. We deduce from (2.9) that the sets $W_{m_{n}} \cap V\left(G_{\alpha}\right)$ are mutually disjoint. Let $m=\sup \left\{m_{n}: n \geq 0\right\}$, the supremum being taken in $L$. By (2), since $\operatorname{cf}(\kappa)>\aleph_{0}$ it follows that $m<l$ and hence $\alpha \in V_{m}$. Let $P$ be a path in $G_{\alpha}$ from $W_{m_{0}} \cap V\left(G_{\alpha}\right)$ to $W_{m} \cap V\left(G_{\alpha}\right)$. Then $P$ meets each $W_{m_{n}} \cap V\left(G_{\alpha}\right)$, which is impossible because there are infinitely many such sets and they are mutually disjoint. This contradiction implies that $\alpha$ does not exist, as required.

We deduce from (3) and (4) that in the first two cases the width of $(L, U)$ is indeed $<\kappa$. For the third case we may therefore assume that $\kappa$ is not a successor cardinal, that is, if $\mu<\kappa$ is a cardinal, then $\mu^{+}<\kappa$. Let $\mu<\kappa$ be such that $(L, U)$ has adhesion $\leq \mu$ at $l$; then for every $l^{\prime} \in L$ with $l^{\prime}<l$ there exist $l_{1}, l_{2} \in L$ such that $l^{\prime} \leq l_{1}<l_{2} \leq l,\left|W_{l_{1}} \cap W_{l_{2}}\right| \leq \mu$ and there is no $l^{\prime \prime} \in L$ with $l_{1}<l^{\prime \prime}<l_{2}$. Then $l_{2} \neq l$, since $l$ has no predecessor, and for the same reason we may choose the pair $l_{1}, l_{2}$ with $l_{1} \neq l^{\prime}$. By (2.9),

$$
\left|\bigcap_{l^{\prime}<l^{\prime \prime}<l} V_{l^{\prime \prime}}\right| \leq\left|\left\{\alpha \in \Lambda: V\left(G_{\alpha}\right) \cap W_{l_{1}} \cap W_{l_{2}} \neq \varnothing\right\}\right| \leq \mu,
$$

and therefore $\left|U_{l}^{-}\right| \leq \mu^{+}<\kappa$, because $U_{l}^{-}$is a monotone union of sets, each of cardinality $\leq \mu$. Hence $(L, U)$ has width $<\kappa$ in the third case as well.

The following corollary of $(2.10)$ will be needed in $\S 4$.

(2.11) Let $\kappa$ be an infinite cardinal and let $(L, W)$ be a linear decomposition of width $<\kappa$ of a graph $G$, and let a subdivision of a tree $T$ be isomorphic to a subgraph of $G$. Then there exists a linear decomposition $(L, U)$ (using the same linear ordering) of $T$ of width $<\kappa$.

Proof. Under the assumption, $T$ is isomorphic to a minor of $G$ in which every node is finite, and therefore the result follows from (2.10).

We denote by $2^{<n}$ the set of all sequences of 0 's and 1's of length $<n$, and let $2^{<\omega}=\bigcup_{n \geq 0} 2^{<n}$.

(2.12) A complete linear ordering $(L, \leq)$ is not scattered if and only if there exists a mapping $F$ from $2^{<\omega}$ into the set of nonempty closed intervals of $L$ such that

(i) if $s^{\prime} \in 2^{<\omega}$ is an extension of $s$, then $F\left(s^{\prime}\right) \subseteq F(s)$, and

(ii) if $s, s^{\prime} \in 2^{<\omega}$ have the same length and $s \neq s^{\prime}$, then $F(s) \cap F\left(s^{\prime}\right)=\varnothing$. 
Proof. The "only if" part is easy (and is not used here). We prove the "if" part as follows. For $s \in 2^{<\omega}$, let $s_{1}, s_{2} \in 2^{<\omega}$ be the two 1-term extensions of $s$, where $\sup \left(F\left(s_{1}\right)\right) \leq \sup \left(F\left(s_{2}\right)\right)$. We define $f(s)=\sup \left(F\left(s_{1}\right)\right)$. It is easy to see that $f$ is an injection. Let $L_{0}=\left\{f(s): s \in 2^{<\omega}\right\}$. We claim that for all $f(s), f\left(s^{\prime}\right) \in L_{0}$ with $f(s)<f\left(s^{\prime}\right)$, there exists $l \in L_{0}$ with $f(s)<l<f\left(s^{\prime}\right)$. For let $s_{1}, s_{2}$ be as before, and define $s_{1}^{\prime}, s_{2}^{\prime}$ similarly for $s^{\prime}$. If $s^{\prime}$ is not an extension of $s$ then $f(s)<f\left(s_{2}\right)<f\left(s^{\prime}\right)$, while if $s$ is an extension of $s^{\prime}$ then $f(s)<f\left(s_{1}^{\prime}\right)<f\left(s^{\prime}\right)$. The claim follows. This is well-known to imply that $L_{0}$ (and hence $L$ ) is not scattered.

(2.13) For each infinite cardinal $\kappa, T_{\kappa}$ does not admit a scattered linear decomposition of width $<\kappa$.

Proof. Let $(L, W)$ be a linear decomposition of $T_{\kappa}$ of width $<\kappa$. We put, for $t \in V\left(T_{\kappa}\right), I_{t}=\left\{l \in L: t \in W_{l}\right\}$. By (L1), (L2), and (L3), each $I_{t}$ is a nonempty closed interval of $L$. We shall construct a mapping $f: 2^{<\omega} \rightarrow V\left(T_{\kappa}\right)$ such that

(i) if $s^{\prime} \in 2^{<\omega}$ is an extension of $s$, then $I_{f\left(s^{\prime}\right)} \subseteq I_{f(s)}$,

(ii) if $s, s^{\prime} \in 2^{<\omega}$ have the same length and $s \neq s^{\prime}$, then $I_{f(s)} \cap I_{f\left(s^{\prime}\right)}=\varnothing$.

Define $f$ of the null sequence to be itself and let us assume that we have already defined $f$ on $2^{<n}$ such that it satisfies (i) and (ii). Let $s \in 2^{<n}-2^{<n-1}$, let $N$ be the set of all neighbours of $f(s)$ in $T_{\kappa}$ and let $a=\inf \left(I_{f(s)}\right), b=$ $\sup \left(I_{f(s)}\right)$. Since $\left|W_{a} \cup W_{b}\right|<\kappa$ it follows that there exists a set $N^{\prime} \subseteq N$ with $\left|N^{\prime}\right|=\kappa$ such that $a, b \notin I_{t}$ and hence $I_{t} \subseteq I_{f(s)}$ for all $t \in N^{\prime}$. If $l \in \bigcap\left\{I_{t}: t \in N^{\prime}\right\}$, then $N^{\prime} \subseteq W_{l}$, contrary to the fact that $(L, W)$ has width $<\kappa$. Hence $\bigcap\left\{I_{t}: t \in N^{\prime}\right\}=\varnothing$ and thus (by completeness) there exist vertices $t_{0}, t_{1} \in N^{\prime}$ such that $I_{t_{0}} \cap I_{t_{1}}=\varnothing$. Let $s_{i}$ be the concatenation of $s$ and $(i)$, and let $f\left(s_{i}\right)=t_{i}(i=0,1)$. This completes the inductive definition of $f$. It follows from the construction that $f$ satisfies (i) and (ii).

Now we put $F(s)=I_{f(s)}$ and it follows that $F$ thus defined satisfies (i) and (ii) of (2.12). Hence $L$ is not scattered, as desired.

The implication (ix) $\Rightarrow$ (i) of (2.3) now follows by combining (2.1)(iii) and (2.13).

\section{ESCAPES OF COUNTABLE ORDER}

In this section we prove (1.2), (1.4), and (1.5). To complete the proof of (1.4) it is enough, by $(2.1)$, to prove the following.

(3.1) $A$ graph is rayless if and only if $<\aleph_{0}$ cops can search it.

Proof. If the graph contains a ray $P$ then the robber can remain uncaptured by staying in a component which contains infinitely many vertices of $P$. For the converse assume that $G$ is rayless. We must describe a winning strategy for the cop player. Let $X_{0}=\varnothing$ and let $R_{0}$ be the robber's response, and assume that $X_{0}, \ldots, X_{n}$ have already been chosen in such a way that $X_{i}=\left\{v_{1}, \ldots, v_{i}\right\}$, where $v_{1}, \ldots, v_{i}$ is a path in $G$, and $v_{i}$ is adjacent to a vertex in $R_{i}$, where $R_{0}, \ldots, R_{n}$ are the robber's responses. Choose a vertex $v_{n+1} \in R_{n}$ adjacent to $v_{n}$ (if $n=0$ choose $v_{1} \in R_{0}$ arbitrarily) and put $X_{n+1}=\left\{v_{1}, \ldots, v_{n}, v_{n+1}\right\}$. It follows that if $R_{n} \nsubseteq X_{n+1}$, then $v_{n+1}$ is adjacent to a vertex in $R_{n+1}$. Since $G$ is rayless there is an $n$ such that $R_{n} \subseteq X_{n+1}$, that is, the cop player wins. 
Our next objective is to prove the full version of (1.5), the following.

(3.2) For a graph $G$, the following conditions are equivalent:

(i) $G$ contains no minor isomorphic to $T_{\aleph_{0}}$,

(ii) $G$ contains no minor isomorphic to $T_{2}$,

(iii) $G$ contains no subgraph isomorphic to a subdivision of $T_{2}$,

(iv) $G$ has no massive escape of order $\aleph_{0}$,

(v) $G$ admits a scattered tree-decomposition of width $<\aleph_{0}$ and adhesion $<\aleph_{0}$,

(vi) $G$ admits a well-ordered decomposition of width $<\aleph_{0}$ and adhesion $<\aleph_{0}$,

(vii) $G$ admits a linear decomposition of width $<\aleph_{0}$ and adhesion $<\aleph_{0}$.

Implications (i) $\Rightarrow$ (ii) $\Rightarrow$ (iii) and (vi) $\Rightarrow$ (vii) are easily seen, and (v) $\Rightarrow$ (vi) follows from (4.6). In this section we prove (iii) $\Rightarrow(\mathrm{iv}) \Rightarrow(\mathrm{v})$ and (vii) $\Rightarrow$ (i). We start with five lemmas.

(3.3) Let $\sigma$ be an escape of order $\kappa \geq \aleph_{0}$ in a graph $G$, let $X, Y \in[V(G)]^{<\kappa}$ and let $C \subseteq \sigma(X)$ be an $X$-flap. Then $C \subseteq \sigma(Y)$ if and only if $C \cap Y=\varnothing$.

Proof. If $C \cap Y \neq \varnothing$ then clearly $C \nsubseteq \sigma(Y)$. So assume that $C \cap Y=\varnothing$. Then $C$ is a $(X \cup Y)$-flap; and hence $C \subseteq \sigma(X \cup Y) \subseteq \sigma(Y)$ by the second and first escape axiom.

Let us recall that if $H$ is a connected subgraph of a graph $G$ and $\sigma$ is an escape in $G$ of order $\kappa$, then $H$ is said to be major if $V(H) \cap(X \cup \sigma(X)) \neq \varnothing$ for every $X \in[V(G)]^{<\kappa}$.

(3.4) Let $G$ be a connected graph, let $\sigma$ be an escape in $G$ of order $\aleph_{0}$, let $P$ be a connected subgraph of $G$, and let $X_{1}, X_{2}, \ldots$ be a sequence of finite subsets of $V(G)$ such that

(i) $X_{i+1} \subseteq X_{i} \cup \sigma\left(X_{i}\right)$ and $\sigma\left(X_{i+1}\right) \subseteq \sigma\left(X_{i}\right)$ for all $i \geq 1$,

(ii) every vertex in $X_{i} \cap X_{i+1}$ is major, for all $i \geq 1$,

(iii) $P$ intersects every $X_{i}(i=1,2, \ldots)$.

Then $P$ is a major subgraph of $G$.

Proof. We first prove the following.

$$
\begin{aligned}
& \text { If } u \in \bigcap_{i \geq 1} \sigma\left(X_{i}\right) \text { is adjacent to } v \in V(G)-\bigcap_{i \geq 1} \sigma\left(X_{i}\right) \text {, then } \\
& v \text { is major. }
\end{aligned}
$$

For $v \notin \sigma\left(X_{i}\right)$ for some $i \geq 1$; hence $v \notin \sigma\left(X_{i+1}\right)$, by (i), and therefore $v \in X_{i} \cap X_{i+1}$, because it is adjacent to $u \in \sigma\left(X_{i}\right) \cap \sigma\left(X_{i+1}\right)$. Hence $v$ is major by (ii).

Suppose, for a contradiction, that $P$ is not major. In particular, no vertex of $P$ is major and so $P$ is infinite by (ii) and (iii). There exists a finite subset $Y^{\prime}$ of $V(G)$ such that $V(P) \subseteq Y^{\prime} \cup C$ for some $Y^{\prime}$-flap $C$ which is disjoint from $\sigma\left(Y^{\prime}\right)$. It follows that $V(P) \cap C \neq \varnothing$ because $P$ is infinite. Let $Y$ be the set of all $v \in Y^{\prime}$ which are adjacent to a vertex in $C$. Then $C$ is a $Y$-flap, and is disjoint from $\sigma(Y)$ by (3.3). Since $C$ is not a subset of $\bigcap_{i \geq 1} \sigma\left(X_{i}\right)$ by (iii), and no vertex of $C$ is major, it follows from (1) that $Y \cap \bigcap_{i>1} \sigma\left(X_{i}\right)=\varnothing$. Since $Y$ is finite, (i) implies that there exists $n$ such that $Y \cap \sigma\left(X_{n}\right)=\varnothing$. Then it follows from (3.3) that $\sigma\left(X_{n}\right) \subseteq \sigma(Y)$ and so $C \cap \sigma\left(X_{n}\right)=\varnothing$. Hence, by (i),

$$
V(P) \cap X_{n+1} \subseteq C \cap\left(X_{n} \cup \sigma\left(X_{n}\right)\right) \cap X_{n+1} \subseteq X_{n} \cap X_{n+1},
$$


and so, by (ii) and (iii), $P$ intersects $X_{n+1}$ in a major vertex, and hence $P$ is major, a contradiction to $\sigma(Y) \cap C=\varnothing$.

(3.5) Let $G$ be a graph, let $\sigma$ be an escape in $G$ of order $\kappa \geq \aleph_{0}$, and let $\kappa^{\prime} \leq \kappa$ be a regular cardinal. Then for every $X \in[V(G)]^{<\kappa^{\prime}}$ there exists $Y \in[V(G)]^{<\kappa^{\prime}}$ such that $Y \subseteq X \cup \sigma(X), \sigma(Y) \subseteq \sigma(X)$ and every vertex in $X \cap Y$ is $\kappa^{\prime}$-major. Proof. For each $v \in X$, let $X_{v} \in[V(G)]^{<\kappa^{\prime}}$ be such that $v \notin X_{v} \cup \sigma\left(X_{v}\right)$ if $v$ is not $\kappa^{\prime}$-major and let $X_{v}=\varnothing$ otherwise. Let $X^{\prime}=X \cup \cup_{v \in X} X_{v}$; then $\left|X^{\prime}\right|<\kappa^{\prime}$ since $\kappa^{\prime}$ is regular. Let $Y$ be the set of all $v \in X^{\prime}$ which are adjacent to a vertex in $\sigma\left(X^{\prime}\right)$.

$$
Y \subseteq X \cup \sigma(X)
$$

For let $y \in Y-X$. Since $y$ is adjacent to a vertex in $\sigma\left(X^{\prime}\right)$, it follows that $y \in \sigma\left(X^{\prime}-\{y\}\right) \subseteq \sigma(X)$ by (3.3) and the first escape axiom.

$$
\sigma(Y) \subseteq \sigma(X)
$$

For every $Y$-flap $C \subseteq \sigma(Y)$ intersects $\sigma\left(X^{\prime}\right)$, by the second escape axiom, and hence $C$ is an $X^{\prime}$-flap. Thus $\sigma(Y)=\sigma\left(X^{\prime}\right)$. But $\sigma\left(X^{\prime}\right) \subseteq \sigma(X)$ by the first escape axiom.

$$
\text { Every vertex in } X \cap Y \text { is } \kappa^{\prime} \text {-major. }
$$

For let $v \in X \cap Y$. Since $v$ has a neighbour in $\sigma\left(X^{\prime}\right) \subseteq \sigma\left(X_{v}\right)$ it follows that $v \in X_{v} \cup \sigma\left(X_{v}\right)$, and so $v$ is $\kappa^{\prime}$-major by definition of $X_{v}$.

The result now follows from (1), (2) and (3).

Let $\sigma$ be an escape of order $\aleph_{0}$ in a graph $G$ and let $X, Y$ be finite subsets of $V(G)$. Recall that $Y$ is later than $X$ if $|Y| \leq|X|, Y \subseteq X \cup \sigma(X)$, and $\sigma(Y) \subseteq \sigma(X)$. This relation is transitive, as is easily seen.

(3.6) Let $G$ be a graph and let $\sigma$ be an escape in $G$ of order $\aleph_{0}$. Let $X, Y$ be finite subsets of $V(G)$ such that $Y \subseteq X \cup \sigma(X)$ and $\sigma(Y) \subseteq \sigma(X)$, and assume that there is no set $X^{\prime} \subseteq V(G)$ later than $X$ with $\left|X^{\prime}\right|<|X|$. Then there exist $|X|$ disjoint paths between $X$ and $Y$.

Proof. Suppose not. Then by Menger's theorem there exists a set $Z \subseteq V(G)$ with $|Z|<|X|$ which intersects every path between $X$ and $Y$. Choose such a set $Z$ with $|Z|$ minimum. Then $Z \subseteq X \cup \sigma(X)$, and since $Z$ is not later than $X$ it follows that $\sigma(X) \nsubseteq \sigma(X)$. By (3.3) there exists a $Z$-flap $C \subseteq \sigma(Z)$ which intersects $X$. Since $Z$ separates $X$ and $Y$ we deduce that $C \cap Y=$ $\varnothing$, and hence $C \subseteq \sigma(Y)$ by (3.3). But $\sigma(Y) \subseteq \sigma(X)$, and $C \cap X \neq \varnothing$, a contradiction.

(3.7) Let $\sigma$ be a massive escape of order $\aleph_{0}$ in a graph $G$, let $Z$ be a finite subset of $V(G)$, and let $D \subseteq \sigma(Z)$ be a Z-flap. Let $T$ be a tree with $|V(T)|=$ $\aleph_{0}$. Then $G$ has a minor isomorphic to $T$ whose nodes are major subgraphs contained in $D$.

Proof. Let the vertices of $T$ be numbered $t_{1}, t_{2}, \ldots$ in such a way that for every $n \geq 1$, exactly one of $t_{1}, t_{2}, \ldots, t_{n}$ is adjacent to $t_{n+1}$. (Such a numbering exists; for let $V(T)=\left\{v_{1}, v_{2}, \ldots\right\}$, let $t_{1}=v_{1}$, and inductively for $i \geq 1$, let $t_{i+1}=v_{j}$ where $j$ is minimum such that $v_{j} \neq t_{1}, \ldots, t_{i}$ and $v_{j}$ 
is adjacent to one of $t_{1}, \ldots, t_{i}$.) Let $k \geq 0$ be an integer. A pair $(\mathscr{C}, X)$ is called a $k$-minority if

(i) $\mathscr{C}=\left(C_{1}, \ldots, C_{k}\right)$ is a sequence of $k$ disjoint finite connected subgraphs of $G$ with $V\left(C_{i}\right) \subseteq D$ for $1 \leq i \leq k$,

(ii) $X \subseteq Z \cup \sigma(Z)$, and $\sigma(X) \subseteq \sigma(Z)$, and $X$ is terminal,

(iii) $V\left(C_{i}\right) \cap X \neq \varnothing$ and $V\left(C_{i}\right) \cap \sigma(X)=\varnothing$ for $1 \leq i \leq k$,

(iv) for $1 \leq i<j \leq k$, if $t_{i}$ and $t_{j}$ are adjacent in $T$, then some edge of $G$ has one end in $V\left(C_{i}\right)$ and the other in $V\left(C_{j}\right)$.

Let $Z^{\prime}$ be a terminal set later than $Z$. Obviously, $\left(\varnothing, Z^{\prime}\right)$ is a 0-minority.

A $k^{\prime}$-minority $\left(\mathscr{C}^{\prime}, X^{\prime}\right)$ is said to extend a k-minority $(\mathscr{C}, X)$ if (writing $\mathscr{C}=\left(C_{1}, \ldots, C_{k}\right)$ and $\left.\mathscr{C}^{\prime}=\left(C_{1}^{\prime}, \ldots, C_{k^{\prime}}^{\prime}\right)\right), k \leq k^{\prime}, C_{i}$ is a subgraph of $C_{i}^{\prime}$ for $1 \leq i \leq k, X^{\prime} \subseteq X \cup \sigma(X)$, and $\sigma\left(X^{\prime}\right) \subseteq \sigma(X)$. A $k^{\prime}$-minority $\left(\mathscr{C}^{\prime}, X^{\prime}\right)$ is said to properly extend a $k$-minority $(\mathscr{C}, X)$ if it extends $(\mathscr{C}, X)$ and every vertex in $X \cap X^{\prime}$ is major.

(2) Let $k \geq 0$ be an integer, and let $(\mathscr{C}, X)$ be a k-minority. Then there exists a $(k+1)$-minority $\left(\mathscr{C}^{\prime}, X^{\prime}\right)$ which properly extends $(\mathscr{C}, X)$.

For let $\mathscr{C}=\left\{C_{1}, \ldots, C_{k}\right\}$ and let $i$ be such that $1 \leq i \leq k$ and $t_{i}$ and $t_{k+1}$ are adjacent. Choose $u \in V\left(C_{i}\right) \cap X$ and let $v \in \sigma(X)$ be a neighbour of $u$, which exists, because otherwise $X-\{u\}$ is later than $X$ by (3.3), contrary to the fact that $X$ is terminal. Since $u \in D$ and $v \notin Z$ we deduce that $v \in D$. (If $k=0$ we choose $v \in \sigma(X) \cap D$ arbitrarily.) Let $Y=X \cup\{v\}$ and let $C_{k+1}$ be the subgraph of $G$ with $V\left(C_{k+1}\right)=\{v\}, E\left(C_{k+1}\right)=\varnothing$. From (3.5) there exists a finite set $Y^{\prime} \subseteq V(G)$ such that $Y^{\prime} \subseteq Y \cup \sigma(Y), \sigma\left(Y^{\prime}\right) \subseteq \sigma(Y)$ and every vertex in $Y^{\prime} \cap Y$ is major. Let $X^{\prime}$ be a terminal set later than $Y^{\prime}$. We have $\sigma(Y) \subseteq \sigma(X)$ from the first escape axiom, and hence

$$
\begin{gathered}
X^{\prime} \subseteq Y^{\prime} \cup \sigma\left(Y^{\prime}\right) \subseteq Y \cup \sigma(Y) \subseteq X \cup \sigma(X) \subseteq Z \cup \sigma(Z), \\
\sigma\left(X^{\prime}\right) \subseteq \sigma\left(Y^{\prime}\right) \subseteq \sigma(Y) \subseteq \sigma(X) \subseteq \sigma(Z) .
\end{gathered}
$$

To finish the proof of (2) we need two more claims.

Every path joining $Z$ and $X^{\prime}$ intersects $X$.

For let $C$ be an $X$-flap. If $C \cap X^{\prime} \neq \varnothing$ then $C \subseteq \sigma(X)$ because $X^{\prime} \subseteq$ $X \cup \sigma(X)$, and if $C \cap Z \neq \varnothing$ then $C \nsubseteq \sigma(X)$ by (ii). Thus no $X$-flap intersects both $Z$ and $X^{\prime}$, and (3) follows.

There exist $|Y|$ disjoint paths between $Y$ and $X^{\prime}$.

For there is no $U$ later than $Y$ with $|U|<|X|+1=|Y|$, because $X$ is terminal. Hence (4) follows from (3.6).

Choose paths as in (4), minimal. Choose $v_{j} \in Y \cap V\left(C_{j}\right)$ for $j=1, \ldots, k+$ 1 , and let $P_{j}$ be that of the paths from (4) which uses $v_{j}$. Since $X^{\prime} \subseteq Y \cup \sigma(Y)$ it follows that $V\left(P_{j}\right) \subseteq Y \cup \sigma(Y)$. It also follows that each $P_{j}$ is contained in $D$ (for $v_{j} \in V\left(C_{j}\right) \subseteq D$, and no other vertex of $P_{j}$ belongs to $X$; and so $V\left(P_{j}\right) \cap Z=\varnothing$ by (3), and hence $V\left(P_{j}\right) \subseteq D$ ) and $P_{j}$ is disjoint from $\sigma\left(X^{\prime}\right)$ (for otherwise by the minimality of $P_{j}$, some $X^{\prime}$-flap included in $\sigma\left(X^{\prime}\right)$ 
would intersect $Y$ ). Let $C_{j}^{\prime}=C_{j} \cup P_{j}$ and let $\mathscr{C}^{\prime}=\left\{C_{1}^{\prime}, \ldots, C_{k+1}^{\prime}\right\}$. If $j, j^{\prime} \in\{1, \ldots, k+1\}$ are distinct then $V\left(C_{j}^{\prime}\right) \cap V\left(C_{j^{\prime}}^{\prime}\right)=\varnothing$ because $V\left(P_{j^{\prime}}\right) \subseteq$ $\sigma(X) \cup\left\{v_{j}\right\}$ and $V\left(C_{j}^{\prime}\right) \cap \sigma(X)=\varnothing$. It follows that $\left(\mathscr{C}^{\prime}, X^{\prime}\right)$ is a $(k+1)$ minority which properly extends $(\mathscr{C}, X)$, thus proving $(2)$.

From (1) and (2) there exist disjoint connected subgraphs $C_{1}, C_{2}, \ldots$ contained in $D$, and finite subsets $X_{1}, X_{2}, \ldots$ of $V(G)$ such that

(a) $X_{i+1} \subseteq X_{i} \cup \sigma\left(X_{i}\right)$ and each vertex in $X_{i} \cap X_{i+1}$ is major,

(b) $\sigma\left(X_{i+1}\right) \subseteq \sigma\left(X_{i}\right)$,

(c) $C_{i}$ intersects every $X_{j}$ for $j=i, i+1, \ldots$

(d) for $1 \leq i<j$, if $t_{i}$ and $t_{j}$ are adjacent in $T$, then some edge of $G$ has one end in $V\left(C_{i}\right)$ and the other in $V\left(C_{j}\right)$.

It follows that $C_{1}, C_{2}, \ldots$ form the nodes of a minor isomorphic to $T$, and every $C_{i}$ is major by (3.4).

The implication (iii) $\Rightarrow$ (iv) of (3.2) follows from the next result.

(3.8) Let $\sigma$ be a massive escape of order $\aleph_{0}$ in a graph $G$, let $Z$ be a finite subset of $V(G)$ and let $C \subseteq \sigma(Z)$ be a Z-flap. Then there exists a subgraph $T$ of $G$ contained in $C$ isomorphic to a subdivision of $T_{2}$ such that every ray of $T$ is a major subgraph.

Proof. By (3.7) we may choose a minor $H$ of $G$ isomorphic to $T_{2}$ in which every node is major. Then it is easy to produce a subgraph $T$ of $G$ isomorphic to a subdivision of $T_{2}$ in such a way that every ray of $T$ intersects infinitely many nodes of $H$. Hence every ray of $T$ is major.

The following corollary will be used in a later paper.

(3.9) Let $\sigma$ be a massive escape of order $\aleph_{0}$ in a graph $G$. Then there exist infinitely many pairwise disjoint major rays in $G$.

Now we begin the proof of the implication (iv) $\Rightarrow(v)$ of (3.2).

(3.10) Let $\kappa$ be an infinite cardinal, and let $\sigma$ be an escape of order $\kappa$ in a graph $G$. Let $\lambda$ be an ordinal and let $\left\{X_{\alpha}\right\}_{\alpha<\lambda}$ be a sequence of subsets of $V(G)$ such that

(i) $X_{\beta} \subseteq X_{\alpha} \cup \sigma\left(X_{\alpha}\right)$ for $\alpha<\beta<\lambda$, and

(ii) $\sigma\left(X_{\beta}\right) \subseteq \sigma\left(X_{\alpha}\right)$ for $\alpha<\beta<\lambda$.

Let $S=\bigcap_{\alpha<\lambda} \sigma\left(X_{\alpha}\right)$. Then there exists a well-ordered decomposition $(\lambda, W)$ of $G \backslash S$ with $W=\left(W_{\alpha}: \alpha \leq \lambda\right)$ such that

(a) $W_{\alpha} \cap W_{\alpha+1}=X_{\alpha}$ for all $\alpha<\lambda$,

(b) $\sigma\left(X_{\alpha}\right)-S=\bigcup_{\alpha<\beta<\lambda} W_{\beta}-W_{\alpha}$ for all $\alpha<\lambda$, and

(c) $W_{\lambda}=\bigcup_{\alpha<\lambda} \bigcap_{\alpha \leq \beta<\lambda} X_{\beta}$.

Proof. We put $H=G \backslash S$, and for $0 \leq \alpha \leq \lambda$,

$$
W_{\alpha}=\bigcap_{\beta<\alpha}\left[X_{\beta} \cup \sigma\left(X_{\beta}\right)\right] \cap\left[V(H)-\sigma\left(X_{\alpha}\right)\right]
$$

with the convention that $\sigma\left(X_{\lambda}\right)=\varnothing$. We must verify that $(\lambda, W)$ satisfies (WO1)-(WO3), and (a), (b), and (c). 
We first verify (WO2). Let $0 \leq \alpha \leq \beta \leq \gamma \leq \lambda$; then

$$
\begin{aligned}
W_{\alpha} \cap W_{\gamma} & \subseteq \bigcap_{\delta<\gamma}\left[X_{\delta} \cup \sigma\left(X_{\delta}\right)\right] \cap\left[V(H)-\sigma\left(X_{\alpha}\right)\right] \\
& \subseteq \bigcap_{\delta<\beta}\left[X_{\delta} \cup \sigma\left(X_{\delta}\right)\right] \cap\left[V(H)-\sigma\left(X_{\beta}\right)\right]=W_{\beta},
\end{aligned}
$$

by (ii).

To verify (WO3) let $\alpha \leq \lambda$ be a limit ordinal, and let $\beta<\alpha$. Then

$$
\begin{aligned}
\bigcap_{\alpha>\gamma>\beta} W_{\gamma} & =\bigcap_{\alpha>\gamma>\beta} \bigcap_{\delta<\gamma}\left[X_{\delta} \cup \sigma\left(X_{\delta}\right)\right] \cap\left[V(H)-\sigma\left(X_{\gamma}\right)\right] \\
& \subseteq \bigcap_{\delta<\alpha}\left[X_{\delta} \cup \sigma\left(X_{\delta}\right)\right] \cap\left[V(H)-\sigma\left(X_{\alpha}\right)\right]=W_{\alpha} .
\end{aligned}
$$

To verify (WO1) we first observe that for $v \in V(H)$, if $\alpha<\lambda$ is the least ordinal such that $v \notin \sigma\left(X_{\alpha}\right)$, then $v \in W_{\alpha}$. Therefore $\bigcup_{\alpha<\lambda} W_{\alpha}=V(H)$. Now let $u, v \in V(H)$ be adjacent and suppose that there is no $\alpha<\lambda$ such that $u, v \in W_{\alpha}$. By (WO2) we may assume that $\alpha<\beta$ for every $\alpha, \beta<\lambda$ such that $u \in W_{\alpha}, v \in W_{\beta}$. Choose $\beta<\lambda$ with $v \in W_{\beta}$, and let

$$
\alpha=\sup \left\{\gamma<\lambda: u \in W_{\gamma}\right\} .
$$

Then $u \in W_{\alpha}$ by (WO3), $v \notin W_{\alpha}$ and $\alpha<\beta$. It follows that $u \notin X_{\alpha} \cup \sigma\left(X_{\alpha}\right)$ (since $u \notin W_{\alpha+1}$ and $v \in \sigma\left(X_{\alpha}\right)$, a contradiction, since $u, v$ are adjacent. This proves (WO1).

To verify (a) we have

$$
\begin{aligned}
W_{\alpha} \cap W_{\alpha+1} & =\bigcap_{\beta<\alpha}\left[X_{\beta} \cup \sigma\left(X_{\beta}\right)\right] \cap\left[V(H)-\sigma\left(X_{\alpha}\right)\right] \\
& \cap\left[X_{\alpha} \cup \sigma\left(X_{\alpha}\right)\right] \cap\left[V(H)-\sigma\left(X_{\alpha+1}\right)\right] \\
& =X_{\alpha},
\end{aligned}
$$

by (i), (ii).

To verify (b) let first $v \in \sigma\left(X_{\alpha}\right)-S$. Then $v \notin \sigma\left(X_{\beta}\right)$ for some $\beta$ with $\lambda>\beta>\alpha$, and we may assume that $\beta$ is the smallest such ordinal. Then $v \in W_{\beta}-W_{\alpha}$. Conversely, for $\lambda>\beta>\alpha$,

$$
W_{\beta}-W_{\alpha} \subseteq\left[\left(X_{\alpha} \cup \sigma\left(X_{\alpha}\right)\right) \cap V(H)\right]-X_{\alpha}=\sigma\left(X_{\alpha}\right)-S .
$$

To verify (c) we have

$$
\begin{aligned}
W_{\lambda} & =\bigcap_{\beta<\lambda}\left[X_{\beta} \cup \sigma\left(X_{\beta}\right)\right]-\bigcap_{\beta<\lambda} \sigma\left(X_{\beta}\right) \\
& =\bigcup_{\alpha<\lambda}\left[\bigcap_{\beta<\alpha}\left(X_{\beta} \cup \sigma\left(X_{\beta}\right)\right) \cap \bigcap_{\alpha \leq \beta<\lambda} X_{\beta}\right]=\bigcup_{\alpha<\lambda} \bigcap_{\alpha \leq \beta<\lambda} X_{\beta},
\end{aligned}
$$

by (i) and (ii).

(3.11) Let $\sigma$ be a nonmassive escape of order $\aleph_{0}$ in a graph $G$. Then there exist a finite set $Y \subseteq V(G)$, a set $S \subseteq \sigma(Y)$ which is the union of some (possibly none) 
but not all $Y$-flaps in $\sigma(Y)$, an integer $k \geq 0$, and a well-ordered decomposition $(\omega, W)$ of $G \backslash S$ such that:

(a) $Y \subseteq X_{i},\left|X_{i}\right|=k$, and there exist $k$ disjoint paths in $G \backslash S$ between $X_{i}$ and $X_{i+1}(i=0,1, \ldots)$, where $X_{i}=W_{i} \cap W_{i+1} \quad(i=0,1, \ldots)$,

(b) $\sigma\left(X_{i}\right)-S=\bigcup_{j>i} W_{j}-W_{i}(i=0,1, \ldots)$,

(c) $W_{\omega}=Y$.

Proof. Let us choose a finite set $X_{0} \subseteq V(G)$ with $\left|X_{0}\right|$ minimum such that there is no later terminal set. There exists a sequence $X_{0}, X_{1}, \ldots$ of distinct subsets of $V(G)$ such that $X_{i+1}$ is later than $X_{i}$ for every $i=0,1, \ldots$.

There is no $Y$ later than $X_{0}$ with $|Y|<\left|X_{0}\right|$.

For otherwise $Y$ would contradict the choice of $X_{0}$. It follows that

$$
\left|X_{i}\right|=\left|X_{i+1}\right| \text { for } i=0,1, \ldots .
$$

Let $k$ be such that $\left|X_{i}\right|=k$ for $i=0,1, \ldots$. Let $Y=\bigcup_{n \geq 0} \bigcap_{i \geq n} X_{i}$; then there exists an $n \geq 0$ such that $Y \subseteq X_{i}$ for every $i \geq n$, and we may assume that $n=0$. Let $S=\bigcap_{i>0} \sigma\left(X_{i}\right)$; it follows that $Y \cap S=\varnothing$ and that if $v \in S$ is adjacent to $u \in V(G)$, then $u \in Y \cup S$ (because if $u \notin \sigma\left(X_{j_{0}}\right)$ then $u \notin \sigma\left(X_{j}\right)$ for every $j \geq j_{0}$, and hence $\left.u \in Y\right)$. Hence $S$ is a union of $Y$-flaps.

$$
\sigma(Y)-S \text { includes a } Y \text {-flap. }
$$

Since $\varnothing \neq X_{1}-X_{0}$ and $X_{1} \subseteq X_{0} \cup \sigma\left(X_{0}\right)$, there exists an $X_{0}$-flap $C^{\prime} \subseteq \sigma\left(X_{0}\right)$ not contained in $S$. Then $C^{\prime} \subseteq C$ for some $Y$-flap $C \subseteq \sigma(Y)$ by the first escape axiom. Since $C \nsubseteq S$ it follows that $C \cap S=\varnothing$, as desired.

(4) There are $k$ disjoint paths in $G$ between $X_{i}$ and $X_{i+1}$ for $i=0,1, \ldots$.

For there is no $Z$ later than $X_{i}$ with $|Z|<\left|X_{i}\right|$, because such a $Z$ would be later than $X_{0}$, contrary to (1). Hence (4) follows from (3.6).

Since $S$ is a union of $Y$-flaps and $Y \subseteq X_{i} \cap X_{i+1}$, we deduce

$$
\begin{aligned}
& \text { There are } k \text { disjoint paths in } G \backslash S \text { between } X_{i} \text { and } X_{i+1} \text { for } \\
& i=0,1, \ldots \text {. }
\end{aligned}
$$

The existence of the desired well-ordered decomposition now follows from (3.10) with $\kappa=\aleph_{0}, \lambda=\omega$.

The following is (iv) $\Rightarrow(\mathrm{v})$ of (3.2).

(3.12) If $G$ has no massive escape of order $\aleph_{0}$, then $G$ admits a scattered tree-decomposition of width $<\aleph_{0}$ and adhesion $<\aleph_{0}$.

Proof. For the purpose of this proof let us call a tree-decomposition normal, if it is scattered and has width $<\aleph_{0}$ and adhesion $<\aleph_{0}$. As in the proof of $(2.1)$ we deduce that

$$
\begin{aligned}
& \text { If } X \subseteq V(G) \text { is finite and every component of } G \backslash X \text { has a normal } \\
& \text { tree-decomposition, then the whole graph does. }
\end{aligned}
$$

Suppose for a contradiction that $G$ is a graph with no massive escape which does not admit a normal tree-decomposition. For each finite set $X \subseteq V(G)$, let 
us define $\sigma(X)$ to be the union of all $X$-flaps $C$ such that the restriction of $G$ to $C$ does not admit a normal tree-decomposition.

$$
\sigma \text { is an escape of order } \aleph_{0} \text {. }
$$

For the first two escape axioms can be verified as in the proof of (2.1), and the third escape axiom follows from the assumption that $G$ does not have a normal tree-decomposition and from (1).

By assumption, $\sigma$ is not massive and therefore there exist a finite set $Y \subseteq$ $V(G)$, a set $S \subseteq V(G)$, and a well-ordered decomposition $(\omega, W)$ of $H=G \backslash S$ as in (3.11). Since $V(H)$ contains at least one $Y$-flap from $\sigma(Y)$ and subgraphs of graphs with normal tree-decompositions also have such decompositions, we deduce

$$
H \text { does not admit a normal tree-decomposition. }
$$

For $i \geq 0$, let $H_{i}$ be the subgraph of $H$ induced by $W_{i}$, and let $X_{i}=$ $W_{i} \cap W_{i+1}$.

(4) Every $H_{i}$ admits a normal tree-decomposition for $i=0,1,2, \ldots$.

For $V\left(H_{i}\right)=W_{i} \subseteq V(G)-\sigma\left(X_{i}\right)$ by (3.11b), and hence (4) follows from (1).

Now let $\left(T^{i}, W^{i}\right)$ be a normal tree-decomposition of $H_{i}$. Let $T$ be the tree obtained by taking the disjoint union of $T^{i}(i=0,1,2, \ldots)$ and joining some vertex of $T^{i}$ to some vertex of $T^{i+1}$ for each $i \geq 0$; and for each $t \in V(T)$ let $U_{t}=W_{t}^{i} \cup X_{i-1} \cup X_{i}$, where $i$ is such that $t \in V\left(T^{i}\right)$ (and where $X_{-1}$ means $\varnothing)$, and let $U=\left(U_{t}: t \in V(T)\right)$.

$(T, U)$ is a normal tree-decomposition of $H$.

We first verify that $(T, U)$ satisfies (W1) and (W2). We have

$$
\bigcup_{t \in V(T)} U_{t} \supseteq \bigcup_{i \geq 0} \bigcup_{t \in V\left(T^{i}\right)} W_{t}^{i}=\bigcup_{i \geq 0} V\left(H_{i}\right)=\bigcup_{i \geq 0} W_{i}=V(H),
$$

where the last equality uses the fact that $W_{\omega} \subseteq W_{0}$, which follows from (a) and (c) of (3.11). Now let $u, v \in V(H)$ be the endpoints of an edge of $H$. Then $u, v \in W_{i}$ for some $i \geq 0$; hence $u, v \in W_{t}^{i}$ for some $i \in V\left(T^{i}\right)$ and thus $u, v \in U_{t}$. Hence $(T, U)$ satisfies (W1). To verify that it satisfies (W2) let $t_{1}, t_{2}, t_{3} \in V(T)$, let $t_{j} \in V\left(T^{i_{j}}\right) \quad(j=1,2,3)$ and let $t_{2}$ lie on the path between $t_{1}$ and $t_{3}$ in $T$. By symmetry we may assume that $i_{1} \leq i_{3}$, and so $i_{1} \leq i_{2} \leq i_{3}$. If $i_{1}=i_{3}$ then $i_{1}=i_{2}=i_{3}=i$ say and

$$
U_{t_{1}} \cap U_{t_{3}}=\left(W_{t_{1}}^{i} \cup X_{i-1} \cup X_{i}\right) \cap\left(W_{t_{3}}^{i} \cup X_{i-1} \cup X_{i}\right) \subseteq W_{t_{2}}^{i} \cup X_{i-1} \cup X_{i}=U_{t_{2}},
$$

and if $i_{1}<i_{3}$ then

$$
\begin{aligned}
U_{t_{1}} \cap U_{t_{3}} & =\left(W_{t_{1}}^{i_{1}} \cup X_{i_{1}-1} \cup X_{i_{1}}\right) \cap\left(W_{t_{3}}^{i_{3}} \cup X_{i_{3}-1} \cup X_{i_{3}}\right) \\
& \subseteq W_{i_{1}} \cap W_{i_{3}} \subseteq X_{i_{1}} \cap X_{i_{i}+1} \cap \cdots \cap X_{i_{3}-1} \subseteq U_{t_{2}} .
\end{aligned}
$$

Thus, $(T, U)$ satisfies (W2). It follows directly from the construction that $T$ is scattered and that $\left|U_{t}\right|<\aleph_{0}$ for every $t \in V(T)$. Now let $t_{0}, t_{1}, \ldots$ be an infinite path of $T$. If there exist integers $m, n \geq 0$ such that $t_{i} \in V\left(T^{n}\right)$ for every $i \geq m$, then $\left|\bigcup_{i=0}^{\infty} \bigcap_{j \geq i} U_{t_{j}}\right|<\aleph_{0}$ and $\liminf _{i \rightarrow \infty}\left|U_{t_{1}} \cap U_{t_{i+1}}\right|<\aleph_{0}$ because $\left(T^{n}, W^{n}\right)$ is a normal tree-decomposition. If such integers $m, n$ do 
not exist then there exist infinitely many integers $i \geq 0$ with the property that $t_{i} \in V\left(T^{j}\right)$ and $t_{i+1} \in V\left(T^{j+1}\right)$ for some integer $j \geq 0$. Then

$$
U_{t_{i}} \cap U_{t_{i+1}} \subseteq W_{j} \cap W_{j+1}=X_{j}
$$

and hence $\liminf _{i \rightarrow \infty}\left|U_{t_{i}} \cap U_{t_{i+1}}\right| \leq k<\aleph_{0}$ and $\left|\bigcup_{i=0}^{\infty} \bigcap_{j \geq i} U_{t_{j}}\right| \leq k<\aleph_{0}$, where $k=\left|X_{0}\right|=\left|X_{1}\right|=\cdots$. This completes the proof of $(5)$.

Now (3) and (5) contradict each other. Thus our assumption that $G$ is a graph with no massive escape which does not admit a normal tree-decomposition is false, and hence no such graph exists.

Next we turn to the implication (vii) $\Rightarrow$ (i) of (3.2).

(3.13) $T_{\aleph_{0}}$ does not admit a linear decomposition of width $<\aleph_{0}$.

Proof. Suppose for a contradiction that $(L, W)$ is a linear decomposition of width $<\aleph_{0}$ of $T=T_{\aleph_{0}}$. For each $t \in V(T)$, let $I(t)=\left\{l \in L: t \in W_{l}\right\}$. Thus each $I(t)$ is a closed interval in $L$. Let $t_{0}$ be the null sequence, and suppose that we have already defined a path of $T$ with vertices $t_{0}, t_{1}, \ldots, t_{n}$ in order, such that $I\left(t_{0}\right) \supseteq I\left(t_{1}\right) \supseteq \cdots \supseteq I\left(t_{n}\right)$. Let $x, y$ be $\inf \left(I\left(t_{n}\right)\right), \sup \left(I\left(t_{n}\right)\right)$ respectively. Now for each neighbour $t$ of $t_{n}$ in $T, I(t) \cap I\left(t_{n}\right) \neq \varnothing$ by (L1), and yet $I(t) \cap\{x, y\} \neq \varnothing$ for only finitely many such $t$, since $W_{x} \cup W_{y}$ is finite. Thus there is a neighbour $t_{n+1}$ of $t_{n}$ (distinct from $t_{n-1}$, if $n \geq 1$ ) such that $I\left(t_{n+1}\right) \subseteq I\left(t_{n}\right)$. This completes the inductive definition of $t_{0}, t_{1}, t_{2}, \ldots$. Since $L$ is complete and $I\left(t_{0}\right) \supseteq I\left(t_{1}\right) \supseteq \ldots$, there exists $l \in L$ such that $l \in I\left(t_{n}\right)$ for all $n \geq 0$, that is, $t_{n} \in W_{l}$ for all $n \geq 0$. But this is impossible since $W_{l}$ is finite, and so there is no such $(L, W)$, as required.

The last implication of (3.2), namely (vii) $\Rightarrow(\mathrm{i})$, now follows from (2.10) and (3.13).

Our next objective is to prove (1.2). We start with a lemma which is a strengthening of (i) $\Rightarrow$ (ii) of (1.4). If $\sigma$ is an escape of order $\aleph_{0}$ in $G$, a ray $P$ in $G$ is $\sigma$-balanced if every subray of $P$ is major.

(3.14) Let $G$ be a graph and $\sigma$ an escape in $G$ of order $\aleph_{0}$. Let $X_{0}$ be a finite subset of $V(G)$ and let $C \subseteq \sigma\left(X_{0}\right)$ be an $X_{0}$-flap. Then there exists $a$ $\sigma$-balanced ray $P$ of $G$ such that $V(P) \subseteq C$.

Proof. There are two cases.

Case I. For every finite $X \subseteq C$, every $\left(X_{0} \cup X\right)$-flap $C^{\prime} \subseteq \sigma\left(X_{0} \cup X\right) \cap C$ contains a major vertex.

Choose a major vertex $v_{0} \in C$ and let $R_{0}$ be the 0 -edge path with $V\left(R_{0}\right)=$ $\left\{v_{0}\right\}$. Inductively. suppose that we have defined distinct major vertices $v_{0}$, $v_{1}, \ldots, v_{n} \in C$ and a path $R_{n}$ from $v_{0}$ to $v_{n}$ with $V\left(R_{n}\right) \subseteq C$, passing through all of $v_{0}, v_{1}, \ldots, v_{n}$ in order. Since $v_{n}$ is major, it has a neighbour in $\sigma\left(X_{0} \cup V\left(R_{n}\right)\right)$, for otherwise $v_{n} \notin X \cup \sigma(X)$ where $X=X_{0} \cup V\left(R_{n}\right)-\left\{v_{n}\right\}$. Let $C_{n}$ be an $\left(X_{0} \cup V\left(R_{n}\right)\right)$-flap in $\sigma\left(X_{0} \cup V\left(R_{n}\right)\right)$ containing a neighbour of $v_{n}$. Since $C_{n} \cap X_{0}=\varnothing$ and $C_{n}$ has a neighbour in $C$ (namely $v_{n}$ ) it follows that $C_{n} \subseteq C$. Thus there is a major vertex $v_{n+1} \in C_{n}$; let $R_{n+1}$ be the concatenation of $R_{n}$ and a path from $v_{n}$ to $v_{n+1}$ contained within $C_{n} \cup\left\{v_{n}\right\}$. This completes the inductive definition. Now $R=R_{0} \cup R_{1} \cup R_{2} \cup \cdots$ is a ray passing through infinitely many major vertices, and hence $R$ is $\sigma$-balanced; and $V(R) \subseteq C$, as required. 
Case II. There exists a finite $X \subseteq C$, and an $\left(X_{0} \cup X\right)$-flap $C^{\prime} \subseteq \sigma\left(X_{0} \cup X\right) \cap C$ which contains no major vertex.

Let $H$ be the subgraph of $G$ induced by $C^{\prime}$; for a finite set $Y \subseteq V(H)$ we define $\tau(Y)=\sigma\left(X_{0} \cup X \cup Y\right) \cap C^{\prime}$. Then $\tau$ is an escape of order $\aleph_{0}$ in $H$ with no major vertex. Let $Y_{0}$ be a nonempty subset of $V(H)$; from (3.5) there exists a sequence $Y_{0}, Y_{1}, \ldots$ of finite subsets of $V(H)$ such that $Y_{i+1} \cup \tau\left(Y_{i+1}\right) \subseteq \tau\left(Y_{i}\right)$ for $i=0,1, \ldots$. If $v \in Y_{i}$ is adjacent to a vertex in $\sigma\left(Y_{i}\right)$, then there exists a path $Q$ joining $v$ to a vertex of $Y_{i+1}$, adjacent to $\sigma\left(Y_{i+1}\right)$, such that $V(Q)-\{v\} \subseteq \sigma\left(Y_{i}\right)-\sigma\left(Y_{i+1}\right)$. (This follows by examining a minimal path within $\{v\} \cup \sigma\left(Y_{i}\right)$ from $v$ to $\sigma\left(Y_{i+1}\right)$.) It follows that there exists a ray $P$, intersecting every $Y_{i}(i=0,1, \ldots)$. By (3.4), $P$ is $\sigma$-balanced.

Now we can prove (1.2), which we restate.

(3.15) Let $G$ be a connected graph and $\kappa$ an infinite cardinal. If $T$ is a subgraph of $G$ which is a $\kappa$-balanced tree, then there exists a unique escape $\sigma$ in $G$ of order $\kappa$ such that

$$
\begin{aligned}
& \text { for every } X \in[V(G)]^{<\kappa} \text { and every } X \text {-flap } C, C \subseteq \sigma(X) \text { if and } \\
& \text { only if }|C \cap V(T)| \geq \kappa .
\end{aligned}
$$

Conversely, for every escape $\sigma$ of order $\kappa$ in $G$ there exists a subgraph $T$ of $G$ which is a $\kappa$-balanced tree and such that $(*)$ holds.

Proof. If $T$ is as stated, then it is easy to verify that $\sigma$ defined by $(*)$ satisfies the escape axioms. Conversely, let $\sigma$ be an escape in $G$ of order $\kappa$. A subgraph $T$ of $G$ which is a tree is called $\sigma$-balanced if $|E(T)| \geq 1$ and for every edge $e$ of $T$, both components of $T \backslash e$ have at least $\kappa$ vertices and are both major subgraphs, unless $\kappa=\aleph_{0}$, in which case $T$ is also permitted to be a $\sigma$-balanced ray (defined earlier). From (2.8) if $\kappa>\aleph_{0}$, or from (3.14) if $\kappa=\aleph_{0}$, we deduce

(1) There exists a $\sigma$-balanced tree.

If $T_{1}, T_{2}$ are $\sigma$-balanced trees, we write $T_{1}<T_{2}$ if $T_{2}$ is not a ray, and either $T_{1}$ is a proper subgraph of $T_{2}$ or $T_{1}$ is a ray with an infinite subray contained in $T_{2}$. By Zorn's Lemma there exists a maximal $\sigma$-balanced tree $T$. We claim that $T$ is as desired. Let $X \in[V(G)]^{<\kappa}$ and let $C \subseteq \sigma(X)$ be an $X$-flap.

$$
\text { If }|V(T) \cap C| \geq \kappa \text { then there exists an edge } e \text { of } T \text { such that }
$$
an infinite component of $T \backslash e$ is contained in $C$.

This follows easily when $T$ is a ray because then $\kappa=\aleph_{0}$. So assume that $T$ is not a ray and suppose that (2) fails. Then for every edge $e$ of $T$ with at least one endpoint in $C$ there exists a path $P_{e}$ in $T$ containing $e$ with endpoints $u, v$ such that $u, v \in X, u, v$ belong to different components of $T \backslash e$ and $P \backslash\{u, v\}$ is contained in $C$. Let $M_{e}=\{u, v\}$. Since there exist $\kappa$ edges as above and $|X|<\kappa$, there exist $\operatorname{cf}(\kappa)$ edges for which $M_{e}$ is the same, and hence there exist two edges, $e$ and $e^{\prime}$ say such that $M_{e}=M_{e^{\prime}}$ and $P_{e} \neq P_{e^{\prime}}$. This is impossible, because $T$ is a tree.

$$
\text { If }|V(T) \cap C| \geq \kappa \text { then } C \subseteq \sigma(X) \text {. }
$$

For let $e$ be as in (2). Every infinite component of $T \backslash e$ is major, and hence $C \subseteq \sigma(X)$.

$$
\text { If } C \subseteq \sigma(X) \text { then }|V(T) \cap C| \geq \kappa .
$$


For suppose that $C \subseteq \sigma(X)$ and $|V(T) \cap C|<\kappa$. Let $Y=X \cup(V(T) \cap C)$; then there exists a $Y$-flap $C^{\prime} \subseteq \sigma(Y) \cap C$. If $\kappa>\aleph_{0}$ then there is a $\kappa$-nova $S$ in $C^{\prime}$ as in (2.8), and if $\kappa=\aleph_{0}$ then there is a $\sigma$-balanced ray $S$ contained in $C^{\prime}$ by (3.14). Let $P$ be a path joining a vertex $t \in V(T)$ to a vertex $s \in V(S)$ and otherwise disjoint from $T \cup S$. If $S$ is a ray we define $S^{\prime}$ to be the path obtained from $S$ by deleting the finite component of $S \backslash s$, and we define $S^{\prime}=S$ otherwise. We define $T^{\prime}$ analogously. Let $T^{\prime \prime}=T^{\prime} \cup P \cup S^{\prime}$; it is easily seen that $T^{\prime \prime}$ is a $\sigma$-balanced tree, contrary to the maximality of $T$ under $<$.

The theorem now follows from (3) and (4).

We give a characterization of massive escapes of order $\aleph_{0}$ in the spirit of (3.15). We omit the proof, because it is similar to that of (3.15), using (3.8) instead of (2.8) or (3.14).

(3.16) Let $G$ be a connected graph and let $\sigma$ be an escape in $G$ of order $\aleph_{0}$. Then $\sigma$ is massive if and only if the tree $T$ from (3.15) can be chosen in such $a$ way that it contains no infinite path $t_{1}, t_{2}, \ldots$, where each $t_{i}$ has valency 2 in $T$.

Finally, we prove a lemma which will be used in a later paper. Let $G$ be a graph and $\sigma$ an escape of order $\aleph_{0}$ in $G$. A finite set $F \subseteq V(G)$ is said to be free if there is no finite set $X \subseteq V(G)$ with $|X|<|F|$ such that $F \cap \sigma(X)=\varnothing$.

(3.17) Let $G$ be a graph and $\sigma$ an escape in $G$ of order $\aleph_{0}$. A set $F \subseteq V(G)$ is free if and only if there is no $X \subseteq V(G)$ later than $F$ with $|X|<|F|$.

Proof. If $F$ is free then there is clearly no such set. Conversely let $F$ be not free and let $X$ be such that

(i) $|X|<|F|$,

(ii) $F \cap \sigma(X)=\varnothing$,

and subject to (i) and (ii),

(iii) $|X|$ is minimum.

We claim that $X$ is later than $F$. From (ii) and (3.3) we deduce that $\sigma(X) \subseteq$ $\sigma(F)$; we must show that $X \subseteq F \cup \sigma(F)$. Let $v \in X$. By (iii), $\sigma(X-\{v\}) \neq$ $\sigma(X)$, and so by (3.3), $v$ is adjacent to some $u \in \sigma(X) \subseteq \sigma(F)$. Hence $v \in F \cup \sigma(F)$, as required.

The following is a strengthening of (3.5) when $\kappa=\aleph_{0}$.

(3.18) Let $G$ be a graph and let $\sigma$ be an escape of order $\aleph_{0}$ in $G$, and let $X$ be a finite subset of $V(G)$. Then there exists a free set $Y \subseteq V(G)$ such that $Y \subseteq X \cup \sigma(X), \sigma(Y) \subseteq \sigma(X)$, and every vertex in $X \cap Y$ is major.

Proof. By (3.5), there exists a finite subset $Y$ of $V(G)$ such that $Y \subseteq X \cup \sigma(X)$, $\sigma(Y) \subseteq \sigma(X)$, and every vertex in $X \cap Y$ is major. Let us choose this $Y$ with $|Y|$ minimum. If $Z$ is later than $Y$ then

$$
X \cap Z \subseteq X \cap(Y \cup \sigma(Y)) \subseteq(X \cap Y) \cup(X \cap \sigma(X))=X \cap Y,
$$

and so there is no $Z$ later than $Y$ with $|Z|<|Y|$. By (3.17), $Y$ is free, as required.

(3.19) Let $G$ be a graph and let $\sigma$ be an escape of order $\aleph_{0}$ in $G$. A finite set $F \subseteq V(G)$ is free if and only if there are $|F|$ disjoint major paths of $G$, each with an end in $F$. 
Proof. Let $H_{1}, \ldots, H_{|F|}$ be disjoint major subgraphs, each intersecting $F$, and let $X \subseteq V(G)$ be such that $|X|<|F|$. Then $X \cap V\left(H_{i}\right)=\varnothing$ for some $1 \leq i \leq|F|$; hence $V\left(H_{i}\right) \subseteq \sigma(X)$ and thus $F \cap \sigma(X) \neq \varnothing$. Hence $F$ is free.

Conversely let $F$ be free. By (3.18) there exists a sequence $X_{0}=F, X_{1}$, $X_{2}, \ldots$ of subsets of $V(G)$ such that $X_{n+1} \subseteq X_{n} \cup \sigma\left(X_{n}\right), \sigma\left(X_{n+1}\right) \subseteq \sigma\left(X_{n}\right)$, every vertex in $X_{n} \cap X_{n+1}$ is major, and each $X_{n}$ is free. Hence $\left|X_{0}\right| \leq\left|X_{1}\right| \leq$ ... From (3.6) there exist disjoint paths $P_{1}, \ldots, P_{|F|}$, each with an end in $F$ and intersecting every $X_{n}(n=0,1, \ldots)$; and from (3.4), each $P_{i}$ is major.

\section{MAJOR ESCAPES}

In this section we shall state and prove an expanded form of (1.6). To state our results for excluding $T_{\kappa}$ as a subdivision we need a more general type of "tree-decomposition", which we now introduce. A well-founded tree is a pair $T=(V(T), \leq)$, where $V(T)$ is an arbitrary nonempty set, whose elements are called the vertices, and $\leq$ is a partial ordering on $V(T)$ such that for every pair $t, t^{\prime} \in V(T)$ their infimum $\inf \left(t, t^{\prime}\right)$ exists and for every $t \in V(T)$ the set $\left\{t^{\prime} \in V(T): t^{\prime} \leq t\right\}$ is well-ordered. The supremum of order types of these sets is called the height of $T$. For $t_{1}, t_{2} \in V(T)$ we define $T\left[t_{1}, t_{2}\right]$ to be the set $\left\{t \in V(T)\right.$ : either $\inf \left(t_{1}, t_{2}\right) \leq t \leq t_{1}$ or $\left.\inf \left(t_{1}, t_{2}\right) \leq t \leq t_{2}\right\}$.

If $R$ is a tree and $r \in V(R)$, let us define $t_{1} \leq t_{2}$ (for $t_{1}, t_{2} \in V(R)$ ) to mean that $t_{1}$ lies on the path between $r$ and $t_{2}$. It is easily seen that $T=(V(R), \leq)$ is a well-founded tree and conversely every well-founded tree of height $\leq \omega$ arises in this way. Moreover, $T\left[t_{1}, t_{2}\right]=R\left[t_{1}, t_{2}\right]$ for every $t_{1}, t_{2} \in V(R)$, as is easily seen. We call $(V(T), \leq)$ the well-founded tree associated with $R$ and $r$. Let $B$ be the well-founded tree associated with $T_{2}$ and $r \in V\left(T_{2}\right)$, where $r$ is the only vertex of $T_{2}$ of valency 2 . We say that a well-founded tree $T$ is scattered if there is no 1-1 and inf-preserving mapping $\psi: V(B) \rightarrow V(T)$. If $T$ is a well-founded tree associated with $R$ and $r$, then $T$ is scattered if and only if $R$ is scattered in the sense introduced earlier.

A well-founded tree-decomposition of a graph $G$ is a pair $(T, W)$, where $T$ is a well-founded tree and $W=\left(W_{t}: t \in V(T)\right)$ satisfies (W1), (W2) and

$$
\begin{aligned}
& \text { if } C \subseteq V(T) \text { is a chain and } c=\sup C \in V(T) \text {, then } \bigcap_{t \in C} W_{t} \subseteq \\
& W_{c} .
\end{aligned}
$$

We say that $(T, W)$ has width $<\kappa$ if

$$
\left|\bigcup_{t \in C} \bigcap_{\substack{t^{\prime} \in C \\ t^{\prime} \geq t}} W_{t^{\prime}}\right|<\kappa \quad \text { for every chain } C \subseteq V(T) .
$$

The tree-decomposition $(T, W)$ is called scattered if $T$ is scattered, and is said to be of height $\alpha$ if $T$ is of height $\alpha$. Let $R$ be a tree, let $r \in V(R)$ and let $T$ be the well-founded tree associated with $R$ and $r$. Then to every treedecomposition $(R, W)$ there corresponds a well-founded tree-decomposition $(T, W)$ of height $\leq \omega$ and vice versa. It is easy to verify that the conditions in the definitions of widths are the same, and hence $(R, W)$ has width $<\kappa$ if and only if $(T, W)$ has width $<\kappa$.

Now we can state the full version of (1.6). 
(4.1) For a graph $G$ and a cardinal $\kappa \geq \aleph_{0}$, the following conditions are equivalent:

(i) $G$ contains no subgraph isomorphic to a subdivision of $T_{\kappa}$,

(ii) $G$ has no major escape of order $\kappa$,

(iii) $G$ admits a scattered well-founded tree-decomposition of height $\leq \operatorname{cf}(\kappa)$ and width $<\kappa$,

(iv) $G$ admits a scattered well-founded tree-decomposition of width $<\kappa$,

(v) $G$ admits a well-ordered decomposition of width $<\kappa$,

(vi) $G$ admits a scattered linear decomposition of width $<\kappa$.

If $\kappa=\aleph_{0}$ then these conditions are moreover equivalent to

(vii) $G$ admits a linear decomposition of width $<\aleph_{0}$,

(viii) $G$ admits a scattered tree-decomposition of width $<\aleph_{0}$.

Implications (iii) $\Rightarrow$ (iv) and (v) $\Rightarrow($ vi) $\Rightarrow$ (vii) are trivial, (vi) $\Rightarrow$ (i) follows from (2.11) and (2.13), and for $\kappa=\aleph_{0}$ (vii) $\Rightarrow$ (i) follows from (2.11) and (3.13). Finally, for $\kappa=\aleph_{0}$ conditions (iii) and (viii) are equivalent by our earlier remark about well-founded tree decompositions of height $\leq \omega$. We now prove (i) $\Rightarrow$ (ii) $\Rightarrow$ (iii) and (iv) $\Rightarrow$ (v).

The implication (i) $\Rightarrow$ (ii) follows from the next result.

(4.2) Let $\kappa$ be an infinite cardinal. If $G$ has a major escape of order $\kappa$, then $G$ contains a subgraph isomorphic to a subdivision of $T_{\kappa}$, such that every vertex of valency $>2$ of this subgraph is major.

Proof. This follows from (2.6) by letting $\mu=\kappa^{+}$and $l(t)=\kappa$ for all $t \in$ $V\left(T_{\kappa}\right)$.

Now we begin the proof of the implication (ii) $\Rightarrow$ (iii) of (4.1).

(4.3) Let $\kappa$ be an infinite cardinal, let $G$ be a connected graph and let $\sigma$ be an escape in $G$ of order $\kappa$, and assume that there is no major vertex. Then there exists a well-ordered decomposition $(\operatorname{cf}(\kappa), W)$ of $G$ such that

(a) $\left|\bigcup_{\beta<\alpha} \bigcap_{\beta<\beta^{\prime}<\alpha}\left(W_{\beta^{\prime}} \cap W_{\beta^{\prime}+1}\right)\right|<\kappa$ for every $\alpha<\operatorname{cf}(\kappa)$,

(b) $\sigma\left(W_{\alpha} \cap W_{\alpha+1}\right)=\bigcup_{\alpha<\beta<\operatorname{cf}(\kappa)} W_{\beta}-W_{\alpha}$, and

(c) $W_{\mathrm{cf}(\kappa)}=\varnothing$.

Proof. Let $\lambda=\operatorname{cf}(\kappa)$ and let $\left\{\kappa_{\alpha}\right\}_{\alpha<\lambda}$ be a nondecreasing sequence of regular cardinals $\leq \kappa$ with supremum $\kappa$. We shall construct a transfinite sequence $\left\{X_{\alpha}\right\}_{\alpha \leq \lambda}$ of subsets of $V(G)$, with $\left|X_{\alpha}\right|<\kappa$ for all $\alpha \leq \lambda$, as follows. Let $X_{0} \subseteq \bar{V}(G)$ with $\left|X_{0}\right|=1$, and assume that $\alpha \leq \lambda$ and $X_{\beta}$ has already been constructed for all $\beta<\alpha$. If $\alpha$ is a successor ordinal, say $\alpha=\beta+1$, we let $X_{\alpha}$ be the set " $Y$ " of (3.5) with $X=X_{\beta}$ and $\kappa^{\prime}$ chosen to be a regular cardinal such that $\left|X_{\beta}\right|<\kappa^{\prime}$ and $\kappa_{\beta} \leq \kappa^{\prime} \leq \kappa$; and if $\alpha$ is a limit ordinal we let $X_{\alpha}=\bigcup_{\beta<\alpha} \bigcap_{\beta \leq \beta^{\prime}<\alpha} X_{\beta^{\prime}}$. Then the following conditions hold:

(1) $\left|X_{\alpha}\right|<\kappa$, for every $\alpha<\lambda$,

(2) $X_{\alpha} \subseteq X_{\beta} \cup \sigma\left(X_{\beta}\right)$ for $\beta<\alpha<\lambda$,

(3) $\sigma\left(X_{\alpha}\right) \subseteq \sigma\left(X_{\beta}\right)$ for $\beta<\alpha<\lambda$, and

(4) for $\beta<\alpha \leq \lambda$, every vertex in $X_{\alpha} \cap X_{\beta}$ is $\kappa_{\beta}$-major.

These conditions are obviously satisfied except for (3) for limit $\alpha$, which we now prove. Let $\alpha$ be a limit ordinal and $\beta<\alpha$, and let $C \subseteq \sigma\left(X_{\alpha}\right)$ be an $X_{\alpha^{-}}$ flap. Let $Y=\bigcup_{\delta<\alpha} X_{\delta}$. Then there exists by the second escape axiom a $Y$-flap 
$C^{\prime} \subseteq \sigma(Y) \cap C$. We claim that no $u \in C^{\prime}$ is adjacent to a $v \in Y-X_{\alpha}$. For otherwise $v \in X_{\delta}-X_{\delta^{\prime}}$ for some $\delta<\delta^{\prime}<\alpha$. Since $u \in C^{\prime} \subseteq \sigma\left(X_{\delta^{\prime}}\right)$ by the first escape axiom, and $v \notin X_{\delta^{\prime}}$, it follows that $v \in X_{\delta} \cap \sigma\left(X_{\delta^{\prime}}\right) \subseteq X_{\delta} \cap \sigma\left(X_{\delta}\right)=\varnothing$, a contradiction. Hence no $u \in C^{\prime}$ is adjacent to a vertex in $Y-X_{\alpha}$. Therefore $C=C^{\prime} \subseteq \sigma(Y) \subseteq \sigma\left(X_{\beta}\right)$ by the first escape axiom, as desired.

(5) $X_{\lambda}=\varnothing$.

For every vertex in $X_{\lambda}$ is $\kappa_{\beta}$-major for every $\beta<\lambda$ by (4), and hence major. But no major vertex exists.

(6) $\bigcap_{\alpha<\lambda} \sigma\left(X_{\alpha}\right)=\varnothing$.

For otherwise there would be some $u \in \bigcap_{\alpha<\lambda} \sigma\left(X_{\alpha}\right)$ adjacent to some $v \notin$ $\bigcap_{\alpha<\lambda} \sigma\left(X_{\alpha}\right)$. Let $\alpha<\lambda$ be such that $v \notin \sigma\left(X_{\alpha}\right)$; then $v \in X_{\beta}$ for all $\beta$ with $\alpha \leq \beta<\operatorname{cf}(\kappa)$, and hence $v \in X_{\lambda}$, contrary to (5).

The result now follows from (1), (2), (3), (5), (6), and (3.10) applied to the sequence $\left\{X_{\alpha}\right\}_{\alpha<\lambda}$.

Now we prove the implication (ii) $\Rightarrow$ (iii) of (4.1).

(4.4) If $G$ has no major escape of order $\kappa$, then $G$ admits a scattered wellfounded tree-decomposition of height $\leq \mathrm{cf}(\kappa)$ and width $<\kappa$.

Proof. For the purpose of this proof, a scattered well-founded tree-decomposition of height $\leq \mathrm{cf}(\kappa)$ and width $<\kappa$ will be called a normal decomposition. Suppose for a contradiction that $G$ is a graph with no major escape of order $\kappa$ which does not admit a normal decomposition. For $X \in[V(G)]^{<\kappa}$, we define $\sigma(X)$ to be the union of all $X$-flaps $C$ such that the restriction of $G$ to $C$ does not admit a normal decomposition. As in (2.1) or (3.12) it can be verified that $\sigma$ is an escape of order $\kappa$ in $G$, and so is not major. Hence there exists a set $X_{0} \in[V(G)]^{<\kappa}$ and a component $H$ of $G \backslash X_{0}$ which contains no major vertex and such that $V(H) \subseteq \sigma\left(X_{0}\right)$. Hence

$H$ does not admit a normal decomposition.

For $X \in[V(H)]^{<\kappa}$, let $\tau(X)$ be the union of all $X$-flaps of $H$ which do not admit a normal decomposition; it follows that $\tau$ is an escape in $H$ with no major vertex. Let $(\operatorname{cf}(\kappa), W)$ be the well-ordered decomposition of $H$ (relative to $\tau$ ) as in (4.3), and for $\alpha<\operatorname{cf}(\kappa)$ let $H_{\alpha}$ be the subgraph of $H$ induced by $W_{\alpha}$.

(2) Every $H_{\alpha}$ admits a normal decomposition.

For $V\left(H_{\alpha}\right)=W_{\alpha} \subseteq V(H)-\tau\left(W_{\alpha} \cap W_{\alpha+1}\right)$ by (4.3b).

Let $\left(T^{\alpha}, U^{\alpha}\right)$ be a normal decomposition of $H_{\alpha}$ and let $t_{\alpha}$ be the least element of $T^{\alpha}(\alpha<\operatorname{cf}(\kappa))$. Let $T$ be the well-founded tree with $V(T)=$ $\bigcup_{\alpha<\operatorname{cf}(\kappa)} V\left(T^{\alpha}\right)$ in which $t \leq t^{\prime}$ if either $t, t^{\prime} \in V\left(T^{\alpha}\right)$ for some $\alpha<\operatorname{cf}(\kappa)$ and $t \leq t^{\prime}$ in $T^{\alpha}$, or $t=t_{\alpha}$ and $t^{\prime} \in V\left(T^{\beta}\right)$ for some $\alpha<\beta<\operatorname{cf}(\kappa)$. We put, for $t \in V\left(T^{\alpha}\right)$,

$$
U_{t}=U_{t}^{\alpha} \cup\left(W_{\alpha} \cap W_{\alpha+1}\right) \cup \bigcup_{\beta<\alpha} \bigcap_{\beta \leq \beta^{\prime}<\alpha}\left(W_{\beta^{\prime}} \cap W_{\beta^{\prime}+1}\right) \subseteq W_{\alpha},
$$

and put $U=\left(U_{t}: t \in V(T)\right)$. By (4.3a), $\left|U_{t}\right|<\kappa$ for every $t \in V(T)$.

$(T, U)$ is a normal decomposition of $H$. 
For it follows directly from the construction that $T$ is a well-founded tree of height $\leq \mathrm{cf}(\kappa)$, and (W1) and (W2) are verified as in (5) of (3.12) (we omit the details). To verify (W3) let $C \subseteq V(T)$ be a chain and let $c=\sup C$. If there exists $r_{0} \in C$ such that $\left\{t \in C: t \geq r_{0}\right\} \subseteq V\left(T^{\alpha}\right)$ for some $\alpha<\operatorname{cf}(\kappa)$ then $\bigcap_{t \in C} U_{t} \subseteq U_{c}$ by the fact that $\left(T^{\alpha}, U^{\alpha}\right)$ satisfies (W3). Otherwise let

$$
\Lambda=\left\{\gamma<\operatorname{cf}(\kappa): C \cap V\left(T^{\gamma}\right) \neq \varnothing\right\},
$$

and let $\alpha=\sup \Lambda, \beta=\min \Lambda$. Then $\beta<\alpha, \alpha<\operatorname{cf}(\kappa)$ (because $C$ has a supremum) and $c \in V\left(T^{\alpha}\right)$. It follows that

$$
\bigcap_{t \in C} U_{t} \subseteq \bigcap_{\gamma \in \Lambda} W_{\gamma^{\prime}} \subseteq \bigcap_{\beta \leq \gamma<\alpha} W_{\gamma} \cap W_{\gamma+1} \subseteq U_{c},
$$

where the second inclusion follows from (WO2) and (WO3). This completes the proof of the fact that $(T, U)$ is a well-founded tree decomposition. Evidently it is scattered. It remains to prove that it has width $<\kappa$. To this end let $C \subseteq V(T)$ be a chain. If there exists $r_{0} \in C$ such that $\left\{t \in C: t \geq r_{0}\right\} \subseteq V\left(T^{\alpha}\right)$ for some $\alpha<\operatorname{cf}(\kappa)$ then $\left|\bigcup_{t \in C} \bigcap_{t^{\prime} \in C, t^{\prime} \geq t} U_{t^{\prime}}\right|<\kappa$ because $\left(T^{\alpha}, U^{\alpha}\right)$ has width $<\kappa$. Otherwise let $\Lambda, \alpha, \beta$ be as above. If $\alpha<\operatorname{cf}(\kappa)$ then $\sup C=t_{\alpha}$ and $\bigcup_{t \in C} \bigcap_{t^{\prime} \in C, t^{\prime} \geq t} U_{t^{\prime}} \subseteq U_{t_{\alpha}}$, while if $\alpha=\operatorname{cf}(\kappa)$ then

$$
\bigcup_{t \in C} \bigcap_{\substack{t^{\prime} \in C \\ t^{\prime} \geq t}} U_{t^{\prime}} \subseteq \bigcup_{\gamma \in \Lambda} \bigcap_{\substack{\gamma^{\prime} \in \Lambda \\ \gamma^{\prime} \geq \gamma}} W_{\gamma^{\prime}} \subseteq W_{\mathrm{cf}(\kappa)}=\varnothing .
$$

In both cases $\left|\bigcup_{t \in C} \bigcap_{t^{\prime} \in C, t^{\prime} \geq t} U_{t^{\prime}}\right|<\kappa$, as desired. This proves (3).

Conditions (1) and (3) contradict each other and therefore no such graph exists, as required.

Let $T=(V(T), \leq)$ be a well-founded tree. A trunk in $T$ is a nonempty subset $P \subseteq V(T)$, totally ordered by $\leq$, such that if $v \in P$ and $u \leq v$ then $u \in P$. Now let $t \in V(T)$. We denote by $b r(t)$ the well-founded tree $\left(\left\{t^{\prime} \in V(T): t^{\prime} \geq t\right\}, \leq\right)$, and if $t, t^{\prime} \in V(T)$ we say that $t^{\prime}$ is a successor of $t$ if $t^{\prime} \neq t, t \leq t^{\prime}$ and there is no $t^{\prime \prime} \in V(T)-\left\{t, t^{\prime}\right\}$ such that $t \leq t^{\prime \prime} \leq t^{\prime}$. Let $P$ be a trunk in $T$ and $t \in P$. We denote by $P(t)$ the set of all $b r\left(t^{\prime}\right)$, where $t^{\prime} \notin P$ is a successor of $t$.

We need Laver's [4] characterization of scattered well-founded trees, as follows. Let $\mathscr{T}_{0}$ be the class of all one-vertex well-founded trees, and for an ordinal $\alpha>0$ let $\mathscr{T}_{\alpha}$ be the class of well-founded trees $T$ not in $\bigcup_{\beta<\alpha} \mathscr{T}_{\beta}$ such that there is a trunk $P$ in $T$ such that $P(t) \subseteq \bigcup_{\beta<\alpha} \mathscr{T}_{\beta}$ for every $t \in P$. There is a unique minimal such $P$, and it is called the spine of $T \in \mathscr{T}_{\alpha}$. Laver's theorem [4] is the following.

(4.5) Let $\mathscr{T}$ be the class of all scattered well-founded trees. Then $\mathscr{T}=\bigcup_{\alpha \in O n} \mathscr{T}_{\alpha}$.

For a scattered well-founded tree $T$, the unique $\alpha$ such that $T \in \mathscr{T}_{\alpha}$ is called the rank of $T$.

The next result yields the implication (iv) $\Rightarrow(\mathrm{v})$ of (4.1).

(4.6) Let $\kappa$ be an infinite cardinal, and let $G$ be a graph.

(i) If $G$ admits a scattered well-founded tree-decomposition of width $<\kappa$, then $G$ admits a well-ordered decomposition of width $<\kappa$. 
(ii) If $G$ admits a scattered tree-decomposition of width $<\kappa$ and adhesion $<\kappa$, then $G$ admits a well-ordered decomposition of width $<\kappa$ and adhesion $<\kappa$.

Proof. (i) Let $(T, W)$ be a scattered well-founded tree-decomposition of $G$ of width $<\kappa$. We proceed by transfinite induction on the rank of $T$. The theorem obviously holds if the rank of $T$ is 0 , so we assume that the rank of $T$ is $\geq 1$ and the theorem holds for all graphs which admit such a decomposition $\left(T^{\prime}, W^{\prime}\right)$ with $T^{\prime}$ of smaller rank.

Let $P$ be the spine of $T$ and let $P=\left\{p_{\alpha}\right\}_{\alpha<\lambda}$, where for $\alpha<\beta<\lambda$, $p_{\alpha} \leq p_{\beta}$ in the ordering of $T$. For $\alpha<\lambda$, let $N_{\alpha}=\left\{t \in V(T): t \geq t^{\prime}\right.$ for a successor $t^{\prime} \notin P$ of $\left.p_{\alpha}\right\}$ and let $G_{\alpha}$ be the subgraph of $G$ induced by the set $\bigcup\left\{W_{t}-W_{p_{\alpha}}: t \in N_{\alpha}\right\}$. By the induction hypothesis each $G_{\alpha}$ is a disjoint union of graphs each of which admits a well-ordered decomposition of width $<\kappa$. Hence $G_{\alpha}$ itself admits a linear decomposition $\left(L^{\alpha}, X^{\alpha}\right)$ of width $<\kappa$ such that $L^{\alpha}$ is a well-ordering. Let $L^{\lambda}$ be a one-element linear ordering, say $L^{\lambda}=\{m\}$. We may assume that all the $L^{\alpha}(\alpha \leq \lambda)$ are mutually disjoint. Let $L$ be the well-ordered sum $L^{1}+L^{2}+\cdots+L^{\lambda}$. Then $L$ is Dedekind complete, and is a well ordering. For $l \in L-\{m\}$, let us define $X_{l}=W_{p_{\alpha}} \cup X_{l}^{\alpha}$, where $\alpha$ is such that $l \in L^{\alpha}$; and let $X_{m}=\bigcup_{\alpha<\lambda} \bigcap_{\alpha \leq \beta<\lambda} W_{p_{\beta}}$. Finally we put $X=\left(X_{l}: l \in L\right)$. We claim that $(L, X)$ is a linear decomposition of $G$ of width $<\kappa$.

Indeed, the statement about width follows immediately, and so it remains to prove that $(L, X)$ satisfies $(\mathrm{L} 1)-(\mathrm{L} 3)$. To verify $(\mathrm{L} 1)$ we have

$$
\begin{aligned}
\bigcup_{l \in L} X_{l} & \supseteq \bigcup_{\alpha<\lambda} \bigcup_{l \in L^{\alpha}} X_{l}^{\alpha} \cup W_{p_{\alpha}}=\bigcup_{\alpha<\lambda} V\left(G_{\alpha}\right) \cup W_{p_{\alpha}} \\
& =\bigcup_{\alpha<\lambda} \bigcup_{t \in N_{\alpha}} W_{t} \cup W_{p_{\alpha}}=\bigcup_{t \in V(T)} W_{t}=V(G) .
\end{aligned}
$$

Now let $u, v$ be endpoints of an edge of $G$. Then $u, v \in W_{t}$ for some $t \in V(T)$ by (W1). There exists an ordinal $\alpha<\lambda$ such that either $t=p_{\alpha}$, in which case $u, v \in X_{l}$ for every $l \in L^{\alpha}$, or $t \geq t^{\prime}$ for a successor $t^{\prime} \notin P$ of $p_{\alpha}$, in which case $u, v \in V\left(G_{\alpha}\right) \cup W_{p_{\alpha}}$ and thus $u, v \in X_{l}^{\alpha} \cup W_{p_{\alpha}} \subseteq X_{l}$ for some $l \in L^{\alpha}$, by (L1) applied to $\left(L^{\alpha}, X^{\alpha}\right)$. This proves (L1).

To verify (L2) let $a<b<c$ in $L$, and let $a \in L^{\alpha}, b \in L^{\beta}, c \in L^{\gamma}$. If $\alpha=\gamma$ then

$$
X_{a} \cap X_{c}=\left(W_{p_{\alpha}} \cup X_{a}^{\alpha}\right) \cap\left(W_{p_{\alpha}} \cup X_{c}^{\alpha}\right) \subseteq W_{p_{\alpha}} \cup X_{b}^{\alpha}=X_{b},
$$

and if $\alpha<\gamma$ then

This verifies (L2).

$$
X_{a} \cap X_{c} \subseteq \bigcup_{\substack{t \geq p_{\alpha} \\ t \geq p_{\alpha+1}}} W_{t} \cap \bigcup_{t \geq p_{\gamma}} W_{t} \subseteq W_{p_{\beta}} \subseteq X_{b} .
$$

To verify (L3) it suffices, since $L$ is a well-ordering, to show that if $a, b \in L$ and $a<b$, and $b$ has no predecessor in $L$ (that is, for all $l<b$ there exists $l^{\prime}$ with $\left.l<l^{\prime}<b\right)$ then $\bigcap_{a \leq l<b} X_{l} \subseteq X_{b}$. Let $a \in L^{\alpha}, b \in L^{\beta}$; then $\alpha \leq \beta$. If there exists $c \in L^{\beta}$ with $c<b$, then

$$
\bigcap_{a \leq l<b} X_{l} \subseteq \bigcap_{c \leq l<b} X_{l}=W_{p_{\beta}} \cup \bigcap_{c \leq l<b} X_{l}^{\beta} \subseteq W_{p_{\beta}} \cup X_{b}^{\beta}=X_{b}
$$


because $b$ has no predecessor in $L^{\beta}$ and $\left(L^{\beta}, W^{\beta}\right)$ satisfies (L3). We assume that there is no such $c$. Thus $b=\min \left(L^{\beta}\right)$, and $\alpha<\beta$. If $\beta$ is a successor ordinal, say $\beta=\gamma+1$, then $\sup \left(L^{\gamma}\right)$ is the predecessor of $b$ in $L$, a contradiction. Thus $\beta$ is a limit ordinal. We claim that $\bigcap_{\alpha<\gamma<\beta} W_{p_{\gamma}} \subseteq X_{b}$. For if $b=m$ then this is true by definition of $X_{m}$; while if $b \neq m$ then $\beta<\lambda$ and

$$
\bigcap_{\alpha<\gamma<\beta} W_{p_{\gamma}} \subseteq W_{p_{\beta}} \subseteq X_{b}
$$

since $(T, W)$ satisfies (W3) and $p_{\beta}=\sup \left\{p_{\gamma}: \alpha<\gamma<\beta\right\}$. This proves our claim that $\bigcap_{\alpha<\gamma<\beta} W_{p_{\gamma}} \subseteq X_{b}$. But

$$
\bigcap_{\alpha \leq l<b} X_{l}=\bigcap_{a<l<b}\left(X_{a} \cap X_{l}\right) \subseteq \bigcap_{\alpha<\gamma<\beta} W_{p_{\gamma}} \subseteq X_{b}
$$

as required. This proves (L3), and hence $(L, X)$ is a linear decomposition of $G$ of width $<\kappa$.

Now $L$ is a well ordering; let its order type be an ordinal $\gamma$, and let $f: \gamma \rightarrow L$ be an order-preserving bijection. Now since $m=\sup (L)$, it follows that $\gamma$ is a successor ordinal, $\gamma=\beta+1$ say. For $0 \leq \alpha \leq \beta$, define $Y_{\alpha}=X_{f(\alpha)}$, and let $Y=\left(Y_{\alpha}: \alpha \leq \beta\right)$. Thus $(\beta, Y)$ is a well-ordered decomposition of $G$ of width $<\kappa$. This proves (i).

(ii) Now let $(T, W)$ be a scattered tree decomposition of $G$ of width < $\kappa$ and adhesion $<\kappa$. We can regard $T$ as a well-founded tree and apply the construction from (i). We claim that the well-ordered decomposition thus produced has adhesion $<\kappa$.

Indeed, let $l \in L$. If $l$ has a predecessor then the required condition follows easily from the fact that $(T, W)$ has width $<\kappa$. If $l$ does not have a precedessor then (since $\lambda \leq \omega$ ) either there exist $l^{\prime}<l$ and $\alpha<\lambda$ such that $l^{\prime \prime} \in L^{\alpha}$ for every $l^{\prime \prime} \in L$ with $l^{\prime} \leq l^{\prime \prime} \leq l$, or $l=m$. In the former case we apply the induction hypothesis, and in the latter case the required condition follows from the fact that $(T, W)$ has adhesion $<\kappa$.

Finally, we mention without proof a variant of (3.15) and (3.16). A tree $T$ is called $T_{\kappa}$-balanced if for every edge $e$ of $T$, both components of $T \backslash e$ contain a subtree isomorphic to a subdivision of $T_{\kappa}$.

(4.7) Let $\kappa$ be an infinite cardinal and let $\sigma$ be an escape in a graph $G$ of order $\kappa$. Then $\sigma$ is major if and only if the tree $T$ in (3.15) can be chosen to be $T_{\kappa}$-balanced.

\section{ESCAPES AND ENDS}

Our objective here is to discuss a natural partial order of the set of escapes of order $\kappa$, and when $\kappa=\aleph_{0}$ to describe a connection with the "ends" of $G$. If $\kappa$ is a cardinal, let $\sigma(X)=\varnothing$ for all $X \in[V(G)]^{<\kappa}$; we call $\sigma$ the non-escape in $G$ of order $\kappa$. It is convenient to augment the set of all escapes of order $\kappa$ by the non-escape of order $\kappa$; thus, we are now really concerned with the functions $\sigma$ satisfying the first two escape axioms. Let us call such a function $\sigma$ a strategy (of order $\kappa$ ). (Thus, the only strategy of order $\kappa$ which is not an escape is the non-escape of order $\kappa$.) 
There is a natural partial ordering of strategies. If $\sigma_{1}, \sigma_{2}$ are strategies of order $\kappa$ in $G$, we say that $\sigma_{1}$ broadens $\sigma_{2}$ if $\sigma_{2}(X) \subseteq \sigma_{1}(X)$ for all $X \in$ $[V(G)]^{<\kappa}$.

(5.1) The broadening partial ordering of strategies of order $\kappa$ is a lattice.

Proof. If $\Sigma$ is a set of strategies of order $\kappa$, we define

$$
\sigma(X)=\bigcup_{\sigma^{\prime} \in \Sigma} \sigma^{\prime}(X) \quad\left(X \in[V(G)]^{<\kappa}\right) .
$$

Then $\sigma$ is a strategy, and is the join of the elements of $\Sigma$. Since there is also a zero element (namely, the non-escape), it follows that the partial ordering is a lattice.

If $R_{1}$ and $R_{2}$ are rays in a graph $G$, we say they are parallel if for every finite $X \subseteq V(G)$, the unique $X$-flap $C$ with $C \cap V\left(R_{1}\right)$ infinite also has infinite intersection with $V\left(R_{2}\right)$. This is an equivalence relation, and its equivalence classes are called the ends of $G$. This concept was introduced by Halin [3].

If $E$ is an end, let us define $\sigma(X)$ to be the unique $X$-flap $C$ with $C \cap V(R)$ infinite for some (and hence every) $R \in E$, for each finite $X \subseteq V(G)$. This function $\sigma$ is an escape of order $\aleph_{0}$, and is a minimal nonzero element (that is, atom) of the lattice of $(5.1)$ for $\kappa=\aleph_{0}$. For any escape $\sigma$ of order $\aleph_{0}$, we denote by $\mathscr{E}(\sigma)$ the set of all ends $E$ such that $\sigma$ broadens the corresponding atom. Thus, an end $E$ belongs to $\mathscr{E}(\sigma)$ if and only if some member of $E$ is $\sigma$-balanced. In general, a set $\mathscr{F}$ of ends is closed if for each end $E \notin \mathscr{F}$ there is a finite $X \subseteq V(G)$ and an $X$-flap $C$ such that $C \cap V(R)$ is infinite for some $R \in E$, and $C \cap V(R)$ is finite for every member $R$ of every end in $\mathscr{F}$. It is easily verified that the collection of all closed sets of ends forms a topology on the set of ends.

(5.2) If $\sigma$ is a strategy of order $\aleph_{0}$ in $G$, then $\mathscr{E}(\sigma)$ is closed.

Proof. Suppose that $E \notin \mathscr{E}(\sigma)$. Then $\sigma$ does not broaden the corresponding atom, and so there is some finite $X \subseteq V(G)$ such that no $X$-flap in $\sigma(X)$ has infinite intersection with any member of $E$. Choose an $X$-flap $C \nsubseteq \sigma(X)$ such that $C \cap V(R)$ is infinite for some $R \in E$; then $C \cap V(R)$ is finite for each member $R$ of each end in $\mathscr{E}(\sigma)$ since $C \nsubseteq \sigma(X)$, and so $\mathscr{E}(\sigma)$ is closed.

(5.3) If $\mathscr{F}$ is a closed set of ends, then there is a strategy $\sigma$ of order $\aleph_{0}$ such that $\mathscr{E}(\sigma)=\mathscr{F}$.

Proof. Let $\sigma$ be the join of the atoms corresponding to the members of $\mathscr{F}$. Then $\sigma$ is a strategy of order $\aleph_{0}$, and $\mathscr{F} \subseteq \mathscr{E}(\sigma)$; it remains to prove equality. Let $E$ be an end not in $\mathscr{F}$. Since $\mathscr{F}$ is closed there is a finite $X \subseteq V(G)$ and an $X$-flap $C$ such that $C \cap V(R)$ is infinite for some $R \in E$, and $C \cap V(R)$ is finite for every member $R$ of every end in $\mathscr{F}$. By definition of $\sigma, C \nsubseteq \sigma(X)$, and so $E \notin \mathscr{E}(\sigma)$, as required.

(5.4) If $\sigma_{1}, \sigma_{2}$ are different strategies of order $\aleph_{0}$, then $\mathscr{E}\left(\sigma_{1}\right) \neq \mathscr{E}\left(\sigma_{2}\right)$.

Proof. Let $X \subseteq V(G)$ be finite with $\sigma_{1}(X) \neq \sigma_{2}(X)$. By symmetry, we may assume that there is an $X$-flap $C \subseteq \sigma_{1}(X)$ with $C \nsubseteq \sigma_{2}(X)$. By (3.14), there is a $\sigma_{1}$-balanced ray $R$ with $V(R) \subseteq C$. Let $E$ be the end with $R \in E$. Then $E \in \mathscr{E}\left(\sigma_{1}\right)$ since $R$ is $\sigma_{1}$-balanced, and $E \notin \mathscr{E}\left(\sigma_{2}\right)$ since $C \nsubseteq \sigma_{2}(X)$. Thus $\mathscr{E}\left(\sigma_{1}\right) \neq \mathscr{E}\left(\sigma_{2}\right)$. 
From (5.2), (5.3), (5.4) we deduce

(5.5) The function $\mathscr{E}$ is a bijection between the set of all strategies of order $\aleph_{0}$ and the set of all closed sets of ends.

Consequently, every strategy of order $\aleph_{0}$ is a join of atoms. (This is also implied by (3.15).)

As a corollary of (3.15) and (5.3) we deduce

(5.6) Let $\mathscr{F}$ be a closed set of ends of a connected graph $G$. Then there is a subgraph $T$ of $G$ which is a tree, such that for each finite $X \subseteq V(G)$ and every $X$-flap $C, C$ includes a ray of $T$ if and only if $C$ includes some member of some end in $\mathscr{F}$.

In particular, if we let $\mathscr{F}$ be the set of all ends, we deduce

(5.7) For every connected graph $G$ there is a spanning tree $T$ such that for every finite $X \subseteq V(G)$ and every $X$-flap $C, C$ includes a ray of $T$ if and only if $C$ includes a ray of $G$.

This is a weak form of an old conjecture of Halin [3], which we have recently shown to be false [7]; the following.

(5.8) Conjecture. For every connected graph $G$ there is a spanning tree $T$ such that for each end $E$ of $G$ there is exactly one end $F$ of $T$ with $F \subseteq E$.

\section{VARIATIONS OF THE GAME}

In this section we investigate what happens to the cops-and-robber game when the robber is invisible. This makes it no longer a full knowledge game, and luck now plays a role. We are concerned with-in which graphs can the cops guarantee to capture a lucky, invisible robber? Another way to view this game, suggested by the referee, is: the cops are required to submit the sequence of cop moves in advance, and the robber will gain knowledge of this sequence before the game begins. Can the robber use this information to survive infinitely long, or can the cop search sequence be constructed so that the robber will be captured at some finite step no matter what he does?

Let us state the game more precisely. Let $G$ be a graph, and let $\kappa$ be a cardinal. We say that $<\kappa$ cops can blindly search the graph $G$ if there exists a sequence $\varnothing=X_{0}, X_{1}, \ldots$ of subsets of $V(G)$ such that

(i) $\left|X_{i}\right|<\kappa$ for every $i \geq 0$,

(ii) for every $i \geq 0$, either $X_{i} \subseteq X_{i+1}$ or $X_{i+1} \subseteq X_{i}$, and

(iii) there is no sequence $R_{0}, R_{1}, \ldots$ of (nonempty) subsets of $V(G)$ such that $R_{i}$ is an $X_{i}$-flap and $R_{i} \cap R_{i+1} \neq \varnothing$ for every $i \geq 0$.

It is shown in [1] that $<k$ cops can blindly search a finite graph $G$ if and only if $G$ has "path-width" $\leq k-2$. To state a result for infinite graphs we need the following definition. Let $(T, W)$ be a tree-decomposition of a graph $G$, and let $t_{0} \in V(T)$. We call the triple $\left(T, t_{0}, W\right)$ a rooted tree-decomposition of $G$. Let $\kappa$ be a cardinal. We say that a rooted tree-decomposition $\left(T, t_{0}, W\right)$ of a graph $G$ is $<\kappa$ narrow if $\mid \bigcup\left\{W_{t}\right.$ : $\left.\operatorname{dist}\left(t, t_{0}\right)=n\right\} \mid<\kappa$ for every integer $n \geq 0$. 
(6.1) Let $G$ be a graph, and let $\kappa$ be a cardinal. Then $<\kappa$ cops can blindly search $G$ if and only if $G$ admits $a<\kappa$ narrow rooted tree-decomposition $\left(T, t_{0}, W\right)$ such that $T$ is rayless.

For if $G$ admits a $<\kappa$ narrow rayless rooted tree-decomposition, then it is easy to show that $<\kappa$ cops can blindly search $G$. Conversely, it can be shown that if $<\kappa$ cops can blindly search $G$, then they can do so "monotonely", that is, the sequence $X_{0}, X_{1}, \ldots$ satisfies $X_{i} \cap X_{k} \subseteq X_{j}$ for all $k \geq j \geq i \geq 0$ (compare to (2.3) of [1]). From this the required tree-decomposition is easy to obtain. We omit further details.

For $\kappa$ infinite we deduce the following two corollaries.

(6.2) Let $\kappa$ be a cardinal with $\operatorname{cf}(\kappa)=\omega$. Then $<\kappa$ cops can blindly search $G$ if and only if $|V(G)| \leq \kappa$ and $<\kappa$ cops can search $G$.

(6.3) Let $\kappa$ be a cardinal with $\mathrm{cf}(\kappa)>\omega$. Then $<\kappa$ cops can blindly search $G$ if and only if $|V(G)|<\kappa$.

There is also a transfinite version of this searching game, as follows. A blind search in $G$ is a transfinite sequence $\left(X_{\alpha}\right)_{\alpha \leq \lambda}$ of subsets of $V(G)$, where $\lambda$ is an ordinal, such that $X_{0}=\varnothing$, for every ordinal $\alpha$ either $X_{\alpha} \subseteq X_{\alpha+1}$ or $X_{\alpha+1} \subseteq X_{\alpha}$, and $X_{\alpha}=\bigcap_{\beta<\alpha} \bigcup_{\beta<\gamma<\alpha} X_{\gamma}$ for every limit ordinal $\alpha \leq \lambda$. Let $\kappa$ be a cardinal. We say that a blind search $\left(X_{\alpha}\right)_{\alpha \leq \lambda}$ has width $<\kappa$ if $\left|X_{\alpha}\right|<\kappa$ for every $\alpha<\lambda$. If $X=\left(X_{\alpha}\right)_{\alpha \leq \lambda}$ is a blind search, we define $B_{0}(X)=V(G)$, and for $\alpha>0$ let $B_{\alpha}(X)$ be the set of all $v \in V(G)$ such that there is a path in $G$ between $v$ and a vertex in $\bigcap_{\beta<\alpha} \bigcup_{\beta<\gamma<\alpha} B_{\gamma}(X)$ avoiding $X_{\alpha}$. We say that $X$ is successful if $B_{\lambda}(X)=\varnothing$, and we say that $<\kappa$ cops can transfinitely blindly search the graph if there exists a successful blind search of width $<\kappa$.

(6.4) Let $\kappa$ be a cardinal. Then $<\kappa$ cops can transfinitely blindly search $G$ if and only if $G$ admits a well-ordered decomposition of width $<\kappa$.

The proof is similar to the proof of (6.1). Again, we omit the details. For infinite $\kappa$ we deduce from (1.6)

(6.5) Let $\kappa$ be an infinite cardinal. Then $<\kappa$ cops can transfinitely blindly search a graph $G$ if and only if $G$ contains no subgraph isomorphic to a subdivision of $T_{\kappa}$.

The reader may perhaps ask what happens to the regular cops-and-robber game if the cops are allowed to search transfinitely. This game turned out to be useful in characterizing graphs with no minor isomorphic to the complete graph of cardinality $\kappa$. See [6].

\section{UNDECIDABLE STRUCTURE THEOREM}

One might expect a structure theorem of the form "a graph contains no minor isomorphic to $T_{\aleph_{1}}$ if and only if it admits a well-founded tree-decomposition $(T, W)$ of width $<\aleph_{1}$, where $T$ contains no minor isomorphic to $T_{\aleph_{1}}$." Such a theorem indeed exists, but only under an additional set theoretic assumption. We now explain this fact.

Let $T$ be a well-founded tree. We say that a set $K \subseteq V(T)$ is convex if for every $t, t^{\prime}, t^{\prime \prime} \in V(T)$ such that $t^{\prime} \in T\left[t, t^{\prime \prime}\right], t, t^{\prime \prime} \in K$ implies $t^{\prime} \in K$. Let $\left\{K_{\alpha}\right\}_{\alpha \in \Lambda}$ be a collection of disjoint convex subsets of $V(T)$; we define $K_{\alpha} \leq K_{\beta}$ 
if $t \leq t^{\prime}$ for some $t \in K_{\alpha}$ and $t^{\prime} \in K_{\beta}$. Then $\left(\left\{K_{\alpha}\right\}_{\alpha \in \Lambda}, \leq\right)$ is a well-founded tree, called a minor of $T$. Since $T_{\aleph_{1}}$ can be regarded as a well-founded tree, the statement " $T$ has a minor isomorphic to $T_{\aleph_{1}}$ " is well defined. The following is implied by (1.3).

(7.1) Let $G$ be a graph. If $G$ contains no minor isomorphic to $T_{\aleph_{1}}$, then $G$ admits a well-founded tree-decomposition $(T, W)$ of width $<\aleph_{1}$, where $T$ contains no minor isomorphic to $T_{\aleph_{1}}$.

The question is about the converse. A well-founded tree $T$ is called a Suslin tree, if $T$ is uncountable, but every chain and every antichain in $T$ is countable. The Suslin hypothesis is the following statement:

(SH) No Suslin tree exists.

It is known that $(\mathrm{SH})$ is independent of $\mathrm{ZFC}$, and even of $\mathrm{ZFC}+\mathrm{CH}$, or $Z F C+\neg C H$. It turns out that the converse to (7.1) depends on (SH), as follows.

(7.2) The converse to (7.1) holds if and only if (SH) is true.

Indeed, the comparability graph of a Suslin tree admits such a tree-decomposition, but contains a minor isomorphic to $T_{\aleph_{1}}$. We omit further details.

\section{ACKNOWLEDGMENT}

We are indebted to the anonymous referee for carefully reading the manuscript and suggesting many improvements.

\section{REFERENCES}

1. D. Bienstock, N. Roberston, P. D. Seymour, and R. Thomas, Quickly excluding a forest, J. Combin. Theory Ser. B 52 (1991), 274-283.

2. R. Halin, Charakterisierung der Graphen ohne unendliche Wege, Arch. Math. 16 (1965), 227-231.

3. _ـ Über unendliche Wege in Graphen, Math. Ann. 157 (1964), 125-137.

4. R. Laver, Better-quasi-ordering and a class of trees, Studies in Foundations and Combinatorics, Adv. Math. Suppl. Stud., vol. 1, Academic Press, Orlando, 1978.

5. N. Robertson and P. D. Seymour, Graph minors. I, Excluding a forest, J. Combin. Theory Ser. B 35 (1983), 39-61.

6. N. Robertson, P. D. Seymour, and R. Thomas, Excluding infinite clique minors (submitted).

7. P. D. Seymour and R. Thomas, An end-faithful spanning tree counterexample, Proc. Amer. Math. Soc. 113 (1991), 1163-1171.

8. __ Graph searching, and a minimax theorem for tree-width, J. Combin. Theory Ser. B (to appear).

Bellcore, 445 South Street, Morristown, New Jersey 07960

E-mail address: pds@bellcore.com

Dimacs Center, Hill Center, Busch Campus, Rutgers University, New Brunswick, NEW JERSEY 08903

School of Mathematics, Georgia Institute of Technology, Atlanta, Georgia 30332

E-mail address: thomas@math.gatech.edu 